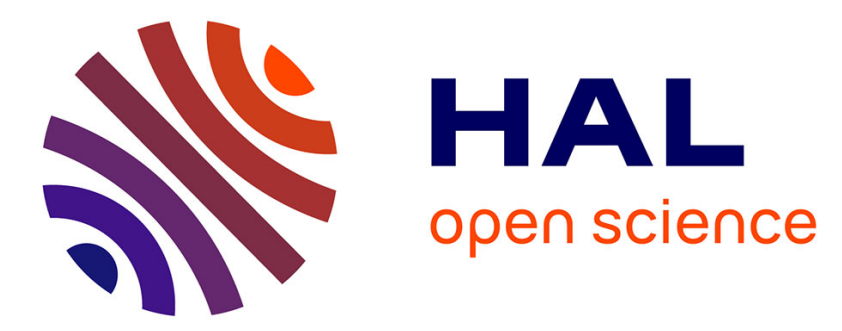

\title{
Vortex control in large-eddy simulations of compressible round jets
}

\author{
M. Maidi, Marcel Lesieur, Olivier Métais
}

\section{To cite this version:}

M. Maidi, Marcel Lesieur, Olivier Métais. Vortex control in large-eddy simulations of compressible round jets. Journal of Turbulence, 2006, 7 (38), pp.1-22. 10.1080/14685240600600410 . hal-00261423

\section{HAL Id: hal-00261423 \\ https://hal.science/hal-00261423}

Submitted on 19 Mar 2020

HAL is a multi-disciplinary open access archive for the deposit and dissemination of scientific research documents, whether they are published or not. The documents may come from teaching and research institutions in France or abroad, or from public or private research centers.
L'archive ouverte pluridisciplinaire HAL, est destinée au dépôt et à la diffusion de documents scientifiques de niveau recherche, publiés ou non, émanant des établissements d'enseignement et de recherche français ou étrangers, des laboratoires publics ou privés. 


\title{
Vortex control in large-eddy simulations of compressible round jets
}

\author{
MOHAMED MAIDI, MARCEL LESIEUR* and OLIVIER MÉTAIS
}

Equipe MoST/LEGI, B.P. 53, 38041 Grenoble Cedex 09, France

\begin{abstract}
We investigate through large-eddy simulations the effects of different types of upstream forcing in subsonic (Mach 0.7) and supersonic (Mach 1.4) round jets. We have reproduced and tested the different methods of forcing developed in incompressible round jets by Urbin and Métais In Direct and LargeEddy Simulations II, 1997, P. R. Chollet, J. P. Voke, and L. Kleiser, Kluwer: Dordrecht, pp. 539-542, Danaila and Boersma, Physics of Fluids A, 12, 1255-1257, da Silva and Métais Physics of Fluids, 14, 3798-3819, (see also Lee and Reynolds Bifurcating and blooming jets at high Reynolds number 5th Symposium on Turbulent Shear Flows, New York). Our strategy is to search the optimal excitation that maximizes the jet spreading at Reynolds number $R e=36000$. Four different forcings based on information obtained both instantaneously and statistically. In the subsonic case, and as in the incompressible one, we aimed to favour the flow spreading along one particular plane (bifurcating plane), while maintaining a standard or reduced spreading rate along the bisecting plane, perpendicular to the bifurcating one. The flow response to the excitations is analysed both instantaneously and statistically. In the subsonic case, and as in the incompressible one, the maximum jet spreading is obtained with inlet varicose-flapping perturbations at preferred and first subharmonic frequencies, respectively. The potential core length is reduced by $27 \%$ with respect to the natural jet. These results are in good agreement with several laboratory experiments and numerical simulations carried out in incompressible round jets. Indeed, the subsonic jet has a convective Mach number of 0.35, and is weakly affected by compressibility. In the supersonic jet case, on the other hand, the highest spreading rate is found with a flapping excitation at the second subharmonic. The potential core length is now reduced by $28 \%$ with respect to the unforced jet.
\end{abstract}

\section{Introduction}

Turbulent jets control represents an important industrial issue today. In aeronautics, the problem due to noise emission at the plane-reactor outlet has led researchers to try to understand its origin, in the hope of reducing the jet acoustic emission. Several previous studies showed that the initial development of the jet has a great influence on noise generation [5-7]. These authors have linked the noise generation mechanism to the shape and dynamics of coherent vortices present in the jet transitional region. In this context, it is of crucial interest to improve the mixing of the jet with the surrounding flow by exciting the large-scale vortices in the upstream region. This can play an important role for jet-noise reduction. Let us mention, for instance, a control method consisting in minimizing acoustic emission by flow characteristics optimization, such as, for example, the mixing rate or the jet transitional zone length.

There are two approaches to jet flow control: active control and passive control. Passive control consists of introducing modifications on the geometry of the nozzle (see [8]). In active

*Corresponding author. E-mail: Marcel.Lesieur@hmg.inpg.fr 
control, the flow is manipulated with the aid of actuators to influence the vortex development, especially in the upstream jet region. In the present study, we are interested in the second type of control.

Various experiments have been carried out to study the reaction of jets to different types of upstream acoustic excitations [4, 9-13]. Using loudspeakers to create several inlet deterministic perturbations, Crow and Champagne [9] found that for a forcing frequency $f$ corresponding to the Strouhal number $\mathrm{St}_{D}=f D / U_{1}=0.3$, the upstream amplitude perturbation reaches its maximum amplitude at the end of the jet potential core $x / D=4$ ( $D$ and $U_{1}$ are the jet diameter and the maximum streamwise velocity at the inlet, respectively). This frequency, called the jet 'preferred mode', corresponds to the passage of approximately toroidal vertical structures at the end of the potential core of free round jets $(4 \leq x / D \leq 6)$.

In order to consider various potential industrial applications, the response of the jet to upstream excitation was studied in detail by Lee and Reynolds [4] with particular interest in the so-called bifurcating jets for the Reynolds number in the range $2.8 \times 10^{3}-1.0 \times 10^{4}$. The bifurcating jet displays a spectacular spreading increase along one particular plane (called bifurcating plane), while keeping a normal or reduced spreading along the plane perpendicular to the bifurcating one (called bisecting plane). With an appropriate forcing combining an axial (varicose) and alternative helical excitation, Lee and Reynolds [4] showed that the jet spreading depends strongly on the ratio of the axial to alternative helical excitation frequencies $r_{f}=$ $f_{a} / f_{h}$. The helical forcing was produced by the orbital motion of the tip of the nozzle. They found that the bifurcating jet is obtained for $r_{f}=2$. The shear-layer in the bifurcating plane takes a $Y$-shaped form. For Reynolds numbers up to 1300, Suzuki et al. [14] experimentally studied the bifurcating jet using flap actuators placed on the side wall of the inlet nozzle. They found that the maximum spreading in the bifurcating plane is obtained when the jet is excited with a frequency corresponding to a Strouhal number $\mathrm{St}_{D}=0.25$. This value represents about half of that measured at the end of potential core of the free jet $\left(\mathrm{St}_{D}=0.52\right)$ for this low Reynolds number. Similar behaviour was noted at high Reynolds numbers $\left(1.0 \times 10^{4} \leq R e \leq\right.$ $\left.1.0 \times 10^{5}\right)$ in the experiments of Parekh et al. [13], using combined axial (varicose) and alternate (flapping) excitation.

The contribution of numerical simulations to this domain is recent. With the aid of largeeddy simulations, Urbin and Métais [1] analysed the response of incompressible round jets to several upstream forcings. They succeeded in numerically reproducing the three types of excitation (varicose, helical and alternate) used in previous experimental studies. By using an alternate excitation, they noted a spectacular spreading of the jet in the bifurcating plane, in a way similar to that observed by Lee and Reynolds [4] and Parekh et al. [13]. Urbin and Métais' alternate forcing consists of imposing a deterministic inlet perturbation causing a speed excess for one half of the jet nozzle and a speed deficit on the other half, and vice versa. The resulting coherent structures exhibit an alternate arrangement as a result of local pairing and also display the $Y$-shaped form. Danaila and Boersma [2] carried out direct numerical simulations at a low Reynolds number $\left(R e=1.5 \times 10^{2}\right)$, and compared the coherent vortices resulting from flapping and bifurcating jets. The flapping jet was obtained with a flapping excitation (alternate excitation) similar to that used by Urbin and Métais [1] for their 'alternate-pairing' excitation. The bifurcating jet resulted from combined varicose (axial) and flapping excitations. The coherent vortices for the flapping excitation are similar to those observed by Urbin and Métais [1]. The jet has a $Y$-shaped form. On the other hand, the bifurcating jet takes a $\Psi$-shaped form. Hilgers [15] also carried out several large-eddy simulations at different Reynolds numbers $\left(1.5 \times 10^{3} \leq R e \leq 1.0 \times 10^{5}\right)$ in order to optimize jet control by favouring the highest possible mixing rate. Like Danaila and Boersma [2], they imposed a combined varicose and flapping inlet excitation. The optimal performances for jet control are obtained when superposing the varicose excitation at the most amplified unstable frequency (preferred 
mode frequency) to the flapping excitation with the same amplitude, but at a subharmonic frequency. More recently, da Silva and Métais [3] studied in detail the role of different inlet perturbations in the vortex dynamics using direct and large-eddy simulations. Their Reynolds number ranges from $R e=1.5 \times 10^{3}$ to $R e=5.0 \times 10^{4}$. In agreement with results of Danaila and Boersma [2], they conclude that the most efficient strategy for jet control is the one combining a varicose excitation at the preferred frequency and a flapping excitation at the subharmonic frequency. Remark that the numerical code they employed was of high order (pseudo-spectral in the transverse directions and sixth-order finite differences in the longitudinal direction), whereas Urbin and Métais' [1] code, developed for industrial applications in complex domains, is of a finite-volume type and much more diffusive numerically. Therefore, it is possible that the latter LES have a lower effective Reynolds number and correspond more to DNS.

It is of crucial interest to assess the capability of these forcings, studied in the incompressible case, to control the subsonic and supersonic round jets, and this is the objective of the present paper. We will rely on information provided by a previous LES of the compressible unforced jet done by Maidi and Lesieur [18].

\section{Numerical details}

In the present numerical investigation, the compressible time-dependent three-dimensional Navier-Stokes and energy equations for air flow are solved in Cartesian cordinates $(x, y, z)$ in the so-called fast-conservation form (details of governing equations can be found in [18]). The molecular Prandtl number is $P r=0.72$ and a perfect gas law is assumed. The functional dependence of viscosity upon temperature is specified through Sutherland's law taking the apropriate constants for air at atmospheric conditions.

The code used is a multi-domain compressible code whose accuracy is a fourth order in space and second order in time. The spatial scheme is Mac Cormack's [19] predictor-corrector scheme, modified by Gottlieb and Turkel [20]. This code was originally developed by Normand and Lesieur [21] and was intensively validated in round jet configurations (see [18], [22]). At each time step, a given velocity profile is prescribed at the inlet, the details of which will be given in the next section. Since we are dealing with spatially evolving compressible flows in which non-physical reflections can be produced at the boundaries, a special treatment of boundary conditions is required. Here, we use a non-reflecting boundary condition proposed by Poinsot and Lele [23]. To absorb outgoing acoustic disturbances and turbulent structures, a sponge zone is placed downstream ([24]) of length $17 \%$ of the computational domain length.

The LES model chosen is the structure function model, originally proposed by Métais and Lesieur [27], in its filtered version [28], where the effects of large-scale inhomogeneities upon the eddy-viscosity are removed by the application of a discretized Laplacien filter to the velocity field. All the simulations presented in this paper are performed with the same computational grid, which has $100 \times 74 \times 74$ points in the streamwise and transverse directions, respectively. The computational domain is a parallelepipedic box of size $35 \times 20 \times 20$ nozzle radii respectively in the longitudinal and transverse directions. In order to correctly simulate the upstream jet shear-layers, the mesh is compressed in the $y$ and $z$ directions with a hyperbolic tangent stretching. Such a discretization $(\approx 550000$ points $)$ is able to cover the streamwise spreading of the jet and allows a well-balanced resolution of the flow field with a reasonable number of grid points. It should be noted that the simulations have been carried out on a regular UNIX workstation. The typical time for the calculation of a fully developed jet is $40 \mathrm{~h}$, which is not excessive. 
Table 1. Large-eddy simulation details presented in this paper.

\begin{tabular}{lccc}
\hline Simulation & Mach & Reynolds & Forcing \\
\hline LES $_{\text {nat1 }}$ & 0.7 & 36000 & Natural \\
LES $_{\text {nat2 }}$ & 1.4 & 36000 & Natural \\
LES $_{\text {for1 }}$ & 0.7 & 36000 & Flap-1 \\
LES for2 $_{\text {LES for3 }}$ & 0.7 & 36000 & Flap-2 \\
LES $_{\text {for4 }}$ & 0.7 & 36000 & A-Flap \\
LES for5 $^{L E S}$ & 1.4 & 36000 & Flap-2 \\
\hline
\end{tabular}

Several LES were carried out (see table 1). Besides the imposed Mach number, the principal differences between the various simulations come from the inlet velocity profile, which is used as an inflow boundary condition. In general, the shape of the inlet velocity profile is

$$
\vec{U}\left(\overrightarrow{x_{0}}, t\right)=\vec{U}_{m}\left(\overrightarrow{x_{0}}\right)+\vec{U}_{\text {forc }}\left(\overrightarrow{x_{0}}, t\right)
$$

It is prescribed at each time step. In equation (1) $\vec{U}=(U, V, W)$, where $U$ is the streamwise velocity component and $V$ and $W$ are the two transverse components. $\overrightarrow{x_{0}}=(x=0, y, z)$ represents the inlet plane. $\overrightarrow{U_{m}}\left(\overrightarrow{x_{0}}\right)=\left(U_{m}, 0,0\right)$ is the mean velocity vector which is given by a hyperbolic tangent profile [25]:

$$
\overrightarrow{U_{m}}\left(\overrightarrow{x_{0}}\right)=\frac{U_{1}+U_{2}}{2}-\frac{U_{1}-U_{2}}{2} \tanh \left[\frac{1}{4} \frac{R}{\theta_{0}}\left(\frac{r}{R}-\frac{R}{r}\right)\right],
$$

where $U_{1}$ is the jet centreline velocity, $U_{2}$ a small coflow and $\theta_{0}$ the momentum thickness of the upstream shear-layer. This profile matches very well with profiles measured in experiments of round jets [26]. Note that the inlet mean transverse velocity components are set to zero:

$$
V_{m}\left(\overrightarrow{x_{0}}\right)=W_{m}\left(\overrightarrow{x_{0}}\right)=0
$$

In order to initiate the turbulence, an additional white noise is imposed on the three upstream velocity components in the shear-layer gradient zone. The maximum intensity of the random noise in our simulations is set to $1.0 \%$ of the inlet maximum velocity. This low intensity allows the jet to evolve naturally. In the excited (forced) jet cases, we impose an upstream aditional forcings $\vec{U}_{\text {forc }}\left(\overrightarrow{x_{0}}, t\right)$ whose detailed formulation will be given in section 4 . Note that in the so-called natural (free) jet, $\vec{U}_{\text {forc }}\left(\overrightarrow{x_{0}}, t\right)=0$.

We take $R / \theta=20$, and the initial centreline and coflow jet velocities are $U_{1}=1.02$ and $U_{2}=0.02$, respectively. The coflow is then very small $\left(U_{2} / U_{1}=0.0196<2 \%\right)$ and does not influence the jet dynamics. The flow is characterized by the jet Mach number $M$, which is defined by means of the upstream jet centre velocity $U_{1}$ and temperature $T_{1}$ :

$$
M=\frac{U_{1}}{\sqrt{\gamma R T_{1}}} .
$$

The Reynolds number is defined with $U_{1}$ and the upstream jet diameter with $D$ :

$$
R e=\frac{U_{1} \rho_{1} D}{\mu_{1}}
$$

$\mu_{1}$ and $\rho_{1}$ being the viscosity and density of the fluid at the jet inflow. 


\section{Natural jet}

In this section, we are interested by vortex dynamics and statistics in a turbulent subsonic and supersonic round jet with only a white noise inlet perturbation. Our goal here is to give reference cases for comparison with the excited jet cases. We stress that a more complete study of natural subsonic and supersonic round jets can be found in detail in [18].

\subsection{Subsonic natural jet}

The first simulation called LES ${ }_{\text {nat } 1}$ concerns a free jet at Mach 0.7 and $R e=36000$. The jet structures are identified using the Q-criterion isosurface technique which is based on the second invariant of the velocity-gradient tensor, $Q=1 / 2\left(\Omega_{i j} \Omega_{i j}-S_{i j} S_{i j}\right), \Omega_{i j}$ and $S_{i j}$ respectively being the antisymmetrical and symmetrical parts of this tensor.

Figure 1 shows instantaneous fields of positive $Q$ coloured by the streamwise vorticity, $\Omega_{x}$ :

$$
\Omega_{x}=\left(\frac{\partial u_{3}}{\partial x_{2}}-\frac{\partial u_{2}}{\partial x_{3}}\right) .
$$

Red or blue corresponds to positive or negative values of $\Omega_{x}$, respectively. One may easily identify upstream the shedding of axisymmetric vortex rings, which is the characteristic of saturation of the Kelvin-Helmholtz instability. These axisymmetric coherent vortices represent the emergence of the so-called varicose mode. As these vortex rings move downstream, they coalesce with neighbouring rings, so that the scale of the vortex rings increases with downstream distance.

We have observed that the axisymmetric vortex rings persist up to $x / D=6$. Further downstream, they grow through an alternate pairing process. This pairing mode corresponds to the growth of a subharmonic perturbation developed after the formation of the primary vortices. This vortex arrangement was also experimentally observed by Broze and Hussain [31] and numerically by Silva and Métais, called varicose-flapping. From $x / D>10$ the coherent structures show a high level of small-scale turbulence, which accelerates the transition towards a three-dimensional turbulence close to isotropy.

The present simulation is compared with incompressible jet measurements carried out within the fully developed region. In this region, round jets obey the following

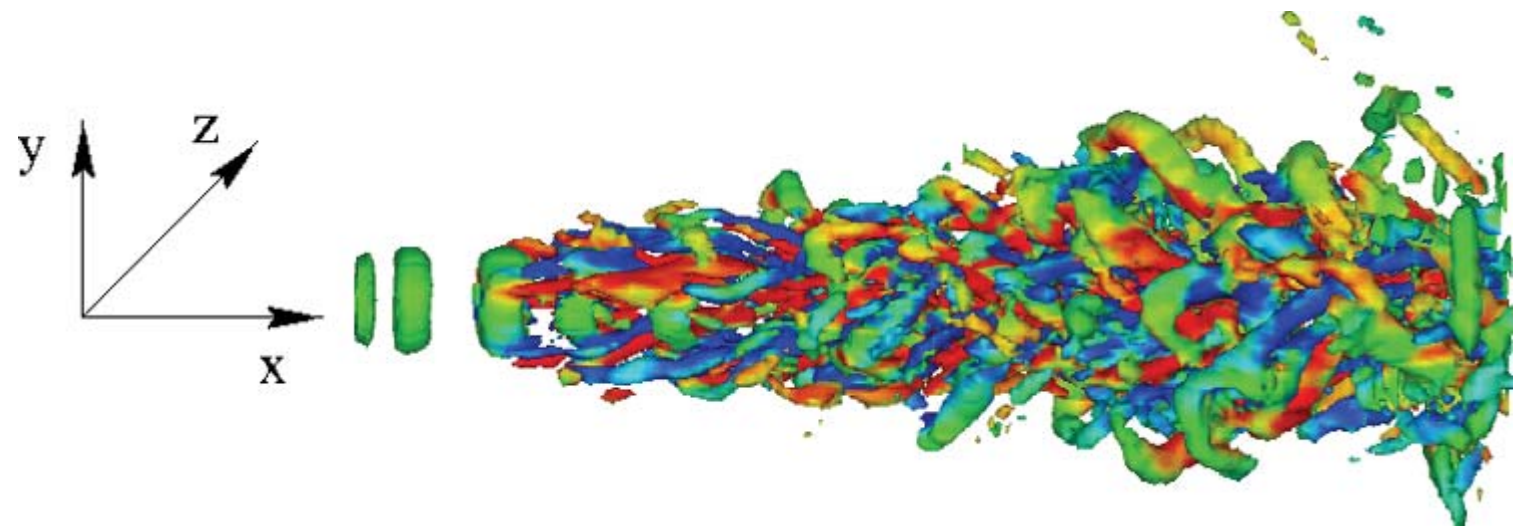

Figure 1. Isosurfaces of positive $Q$ (in green) coloured by the streamwise vorticity $\Omega_{x}$ (positive, red; negative, blue) for the subsonic jet at $M=0.7$ and $R e=36000$. The threshold is $0.20\left(U_{1} / D\right)^{2}$. 


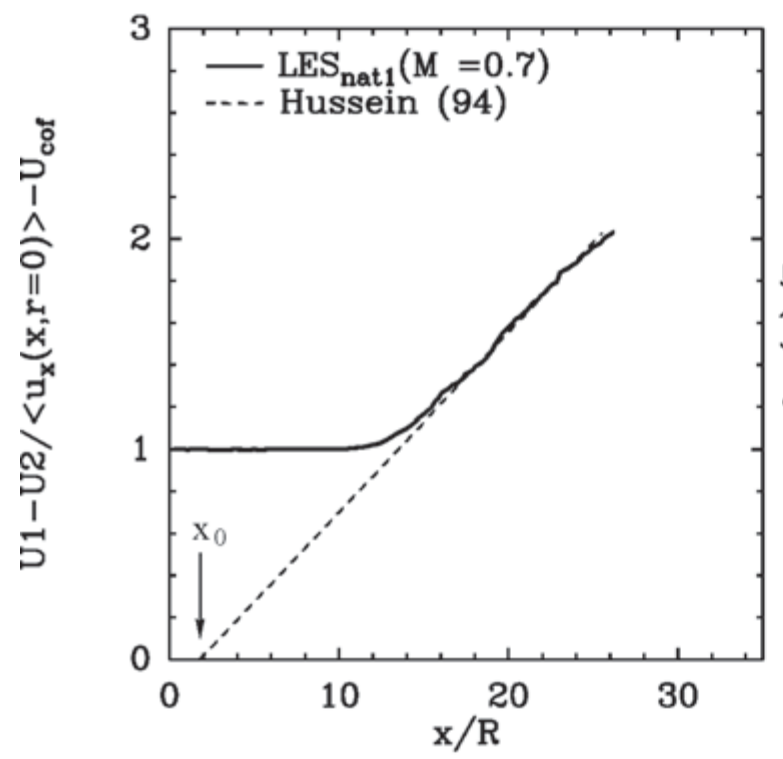

(a)

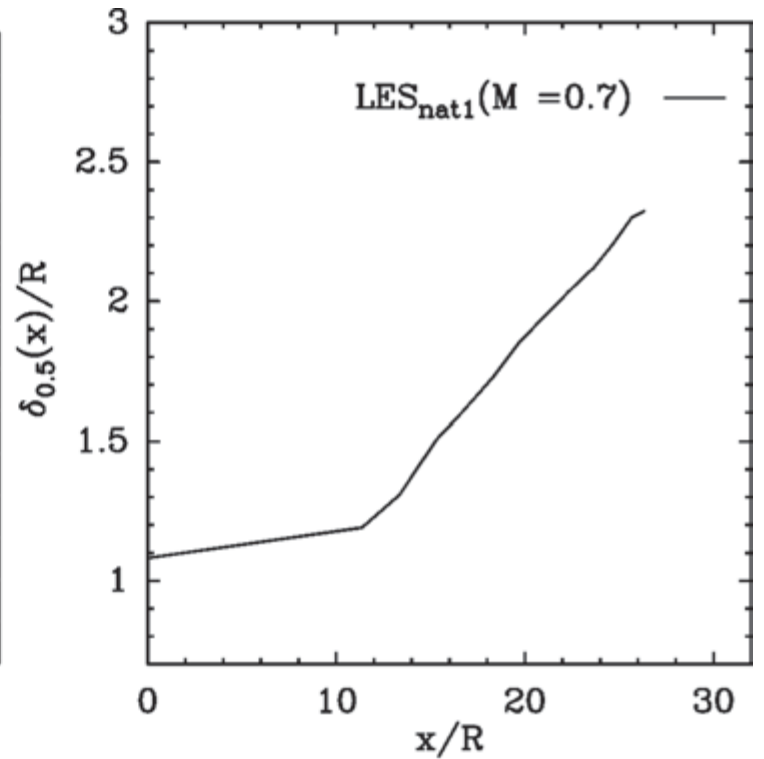

(b)

Figure 2. (a) Streamwise evolution of the centerline velocity decay, (b) streamwise evolution of the jet half-width. $U_{\text {cof }}$ is the local coflow velocity and $x_{o}$ is the virtual origin.

relations [16]:

$$
\begin{aligned}
\frac{U_{1}-U_{2}}{\left\langle u_{x}(x, r=0)-U_{\mathrm{cof}}\right\rangle} & =\frac{1}{B_{u}}\left[\frac{x-x_{0}}{D}\right], \\
\frac{\delta_{0.5}(x)}{D} & =C_{d}\left[\frac{x-x_{0}}{D}\right],
\end{aligned}
$$

$\delta_{0.5}(x)$ is the jet half-width,which is defined as the distance from the jet centreline at which the mean velocity excess equals the mean centreline velocity excess,

$$
\left\langle u_{x}\left(x, r=\delta_{0.5}(x)\right)\right\rangle-U_{\text {cof }}=0.5\left(\left\langle u_{x}(x, r=0)\right\rangle-U_{2}\right) .
$$

$U_{\text {cof }}=\left\langle u_{x}(x, r=\infty)\right\rangle$ is the local coflow and $U_{2}$ is the inlet coflow. The brackets \langle\rangle denote a time average and $x_{0}$ the virtual origin of the jet (see figure 2(a)).

The convective Mach number considered here is about 0.35 , which is well below the value of 0.6 from which the compressibility effects start being important [32], [33]). Figures 2(a) and (b) show the downstream evolution of streamwise velocity and jet half-width, respectively. Both curves show a linear evolution typical of the fully developed turbulent regime. The decay of the centerline mean velocity that occurs at $x / R=11$ corresponds to the end of the potential core. Further downstream, the slope of the centerline velocity decay agrees well with the experimental data of Hussein et al. [16]. To confirm this point, we have computed the centerline velocity decay rate $1 / B_{u}$, in the far field region ( between $x / R=14$ and $x / R=28$ ). We obtain $B_{u}=5.62$, which is very close to the value of $B_{u}=5.8$, obtained by Hussein et al. [16].

Finally, temporal spectra of the longitudinal velocity at Mach 0.7 taken on the centerline at the end of the potential core exhibit a peak frequency at $f_{0} D / U_{1}=0.39$ (figure 3 ). This peak corresponds to the passage frequency of vortices at the end of the potential core. The present value is in good agreement with the typical values found experimentally for the natural round jets $\left(0.3 \leq f_{0} D / U_{1} \leq 0.5,[34]\right)$. This is the preferred mode Strouhal number $\left(\mathrm{St}_{D}^{0}\right)$ which will be used in the following section to simulate the forced jets. 


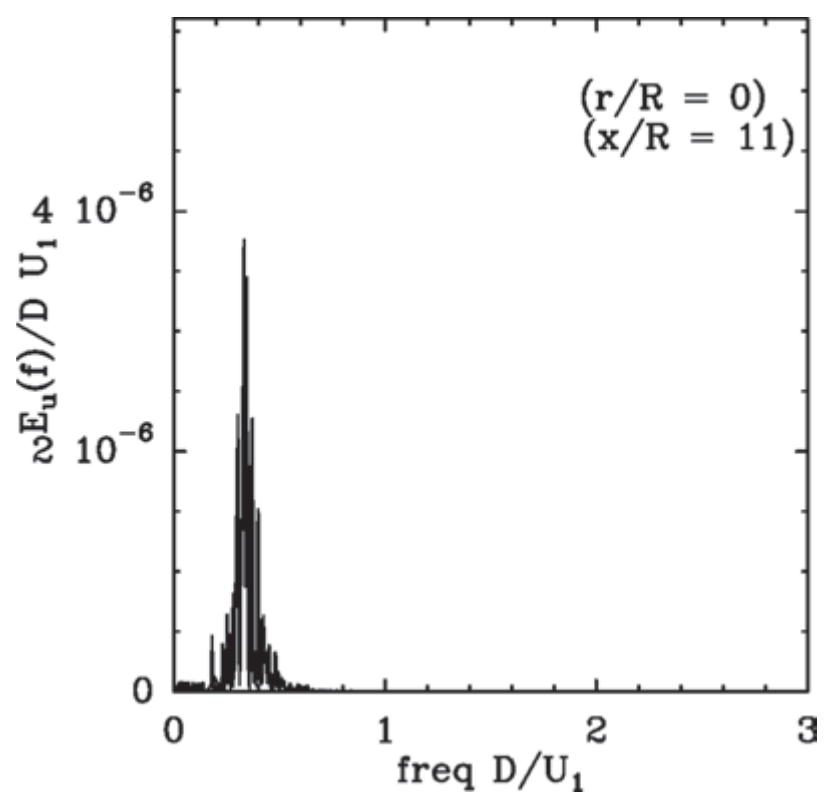

Figure 3. Temporal spectra of the streamwise velocity for the natural jet at $M=0.7$ and $\operatorname{Re}=36000$.

\subsection{Supersonic natural jet}

We study now the supersonic jet at Mach 1.4 and Reynolds 36000. Figure 4 shows that the vortex rings shed from the nozzle at Mach 0.7 disappear. Instead, we have shown that the primary vortices undergoing alternate pairing form immediately. This phenomenon is associated with a dramatic initial reduction of the jet spreading rate, yielding a 27\% increase of the jet potential core length with respect to the subsonic case (figures 5(a) and (b)). Further downstream, the jet has become subsonic and spreads out as in the Mach 0.7 case.

In this case, the preferred mode Strouhal number which will be used after in-forced jet simulations is $\mathrm{St}_{D}^{0}=0.44$ (figure 6). This value corresponds to the preferred mode frequency $\left(f_{0}\right)$ of the natural supersonic jet.

\section{Forced jets}

\subsection{Forcing types}

Previous large-eddy simulations of the subsonic natural jet highlighted upstream shedding of axisymmetric vortex rings, which result from a primary instability of the upstream shear-layer and correspond to a jet preferred mode. From the end of the potential core, these axisymmetric
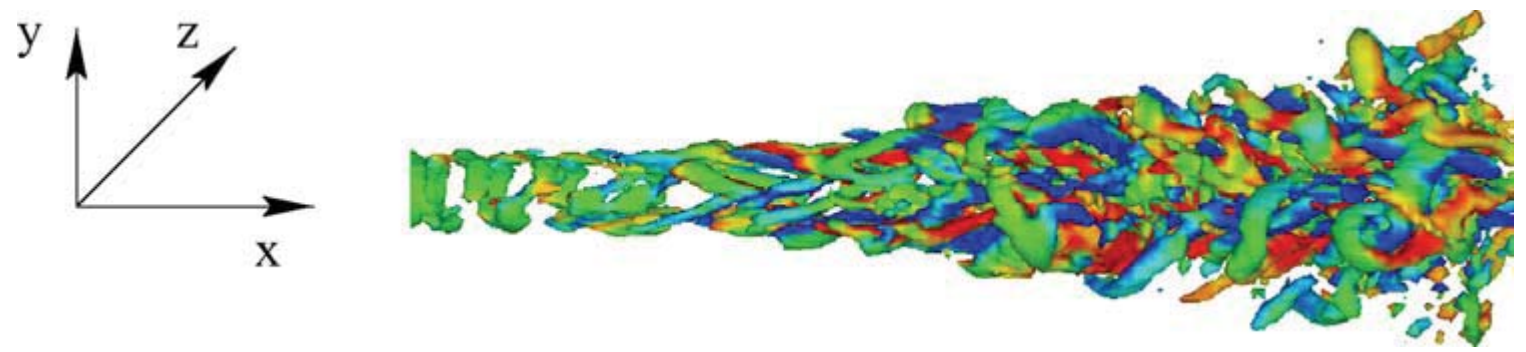

Figure 4. Isosurfaces of positive $Q$ (green) and longitudinal vorticity (red and blue) for the supersonic jet at $M=1.4$ and $R e=36000$. The threshold is $0.20\left(U_{1} / D\right)^{2}$. 


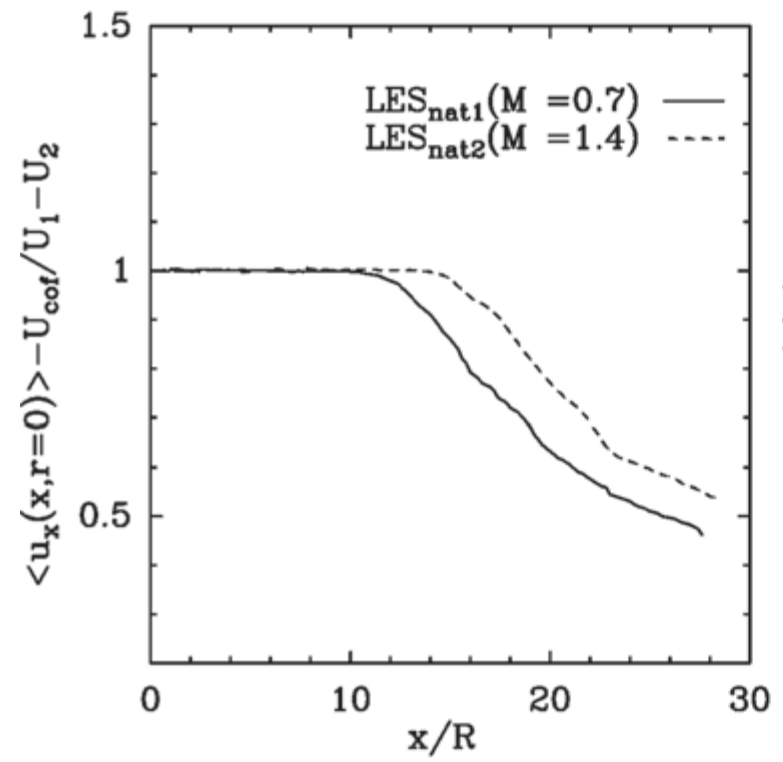

(a)

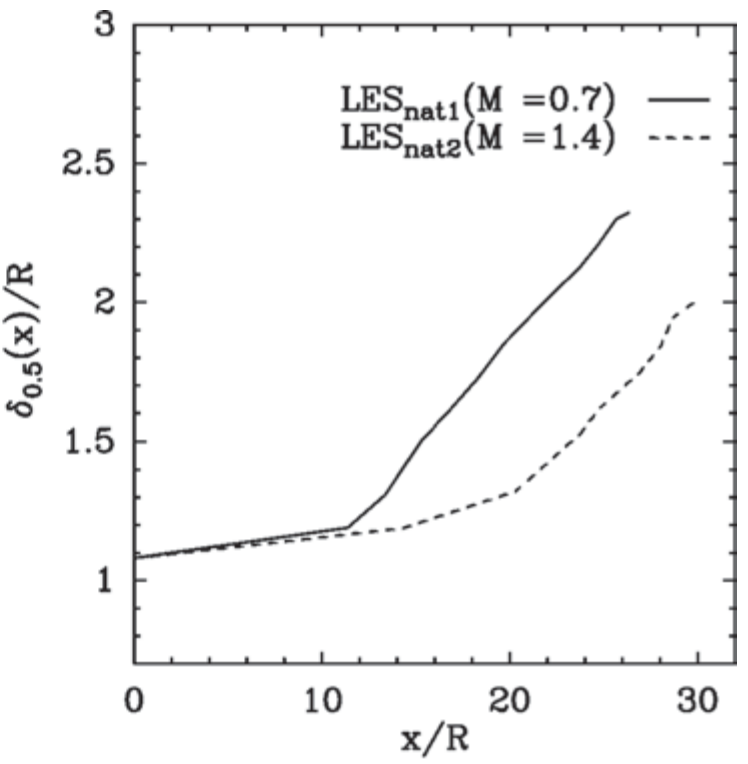

(b)

Figure 5. (a) Streamwise evolution of the centerline velocity decay, (b) streamwise evolution of the shear-layer thickness. $U_{\text {cof }}$ is the local coflow velocity.

vortices develop alternate pairing, resulting from the growth of a subharmonic mode. We have used these two instability modes to carry out a specific strategy of forcing by destabilizing the upstream large-scale vortices along a certain bifurcating plane in order to obtain an important increase of the spreading rate in this plane. Two types of forcing are considered here. The first excitation, named flapping excitation, is similar to that employed by Urbin and Métais [1], Danaila and Boersma [2] and Hilgers [15]. The second excitation, called varicose-flapping, is a superposition of varicose and alternate excitation. This method of forcing has been used by Danaila and Boersma [2], and Silva and Métais [3], and Hilgers [15].

Remark that in some of the previous experimental and numerical studies (for example, [2, $4,10])$, the relative amplitude of the perturbation with respect to the inlet velocity is of the

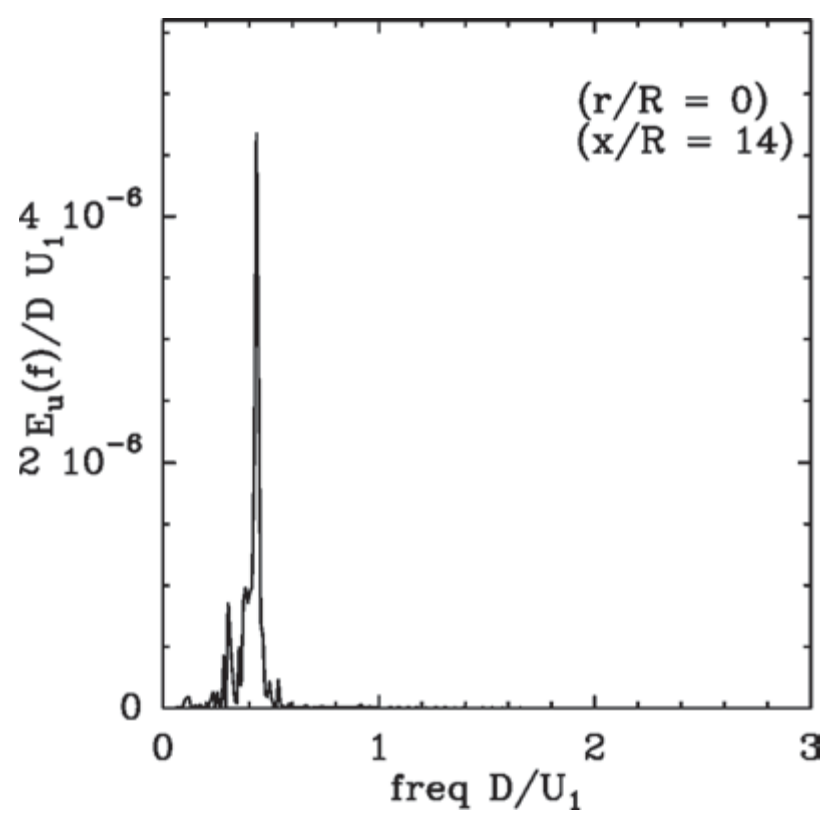

Figure 6. Temporal spectra of the streamwise velocity for the natural jet at $M=1.4$ and $\operatorname{Re}=36000$. 


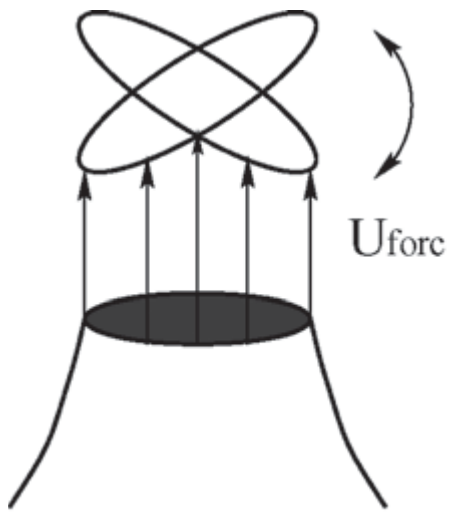

(a)

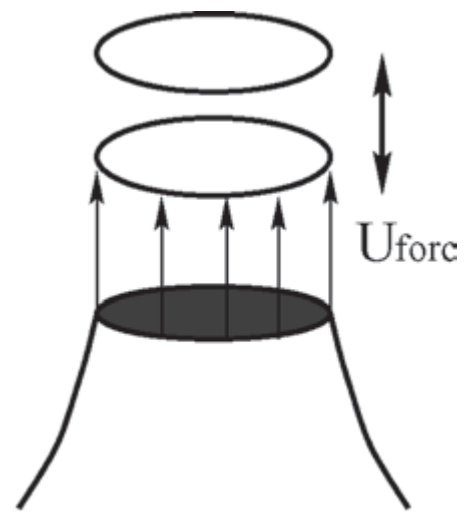

(b)

Figure 7. (a) Sketch of the flapping excitation, (b) sketch of the varicose excitation.

order of $15-20 \%$. In the present study, this relative amplitude is $\epsilon=5 \%$. This is more realistic in terms of potential industrial applications.

4.1.1 Flapping excitation. The flapping excitation is aimed at triggering tilted coherent vortex rings since we have seen that this arrangement corresponds to a jet widening. The corresponding perturbation is

$$
U_{\text {forc }}\left(\overrightarrow{x_{0}}, t\right)=\epsilon U_{1} \sin \left(2 \pi \frac{\mathrm{St}_{D}^{0}}{\alpha} \frac{U_{1} t}{D}\right) \sin \left(\frac{2 \pi y}{D}\right) .
$$

This forcing consists of a combination of a time periodic perturbation at the frequency $f_{o} / \alpha$ and a sinusoidal perturbation along the $y$ direction. Indeed, each half of the jet $(y>0$ or $y<0)$ presents a streamwise velocity excess or deficit, compared to the other part (see figure 7(a)). As mentioned in previous works of Danaila and Boersma [2] and Silva and Métais [3], the plane containing the jet axis and the $y$ direction will be called the bifurcating plane. The plane perpendicular to the bifurcation plane and containing the jet axis will also be called the bisecting plane.

4.1.2 Varicose-flapping excitation. The next excitation is similar to Danaila and Boersma's [2] so-called bifurcating excitation. It is a combination of varicose and flapping excitations. The varicose excitation is obtained by imposing a periodic perturbation to the streamwise velocity at the inlet nozzle through the $U_{m}\left(\overrightarrow{x_{0}}\right)$ profile. This is equivalent to the excitation produced by loudspeakers in experimental works of Crow and Champagne [9], Zaman and Hussain [17]. This excitation (varicose excitation) is aimed at obtaining more intense vortex rings. Figure 7(b) describes schematically this varicose excitation.

The final varicose-flapping forcing expression is given by

$$
\begin{aligned}
U_{\text {forc }}\left(\overrightarrow{x_{0}}, t\right)= & \epsilon U_{m}\left(\overrightarrow{x_{0}}\right) \sin \left(\frac{2 \pi \mathrm{St}_{D}^{0} U_{1} t}{D}\right) \\
& +\epsilon U_{m}\left(\overrightarrow{x_{0}}\right) \sin \left(2 \pi \frac{\mathrm{St}_{D}^{0}}{\alpha} \frac{U_{1} t}{D}+\frac{\pi}{4}\right) \cos (\theta)\left(\frac{2 r}{D}\right),
\end{aligned}
$$

where $r=\sqrt{y^{2}+z^{2}}$ is the radial distance from the jet axis and $\theta$ is the azimuthal angle. 
Table 2. Summary of the several excitation types.

\begin{tabular}{ll}
\hline Forcing & Description \\
\hline Flap-1 & Flapping excitation at preferred mode \\
Flap-2 & Flapping excitation at first subharmonic mode \\
Flap-3 & Flapping excitation at second subharmonic mode \\
A-Flap & Varicose excitation at preferred mode + \\
& flapping excitation at first subharmonic mode \\
\hline
\end{tabular}

Note that almost all the excitations concern only the streamwise velocity component:

$$
V_{\text {forc }}\left(\overrightarrow{x_{0}}, t\right)=W_{\text {forc }}\left(\overrightarrow{x_{0}}, t\right)=0 .
$$

\subsection{Subsonic jet control}

4.2.1 Flapping excitation at the fundamental. The first simulation $\mathrm{LES}_{\text {for } 1}$, which uses forcing Flap-1 (see table 2 for the description of various runs), was carried out with a Strouhal number $\mathrm{St}_{D}^{0}=0.39$ and $\alpha=1$. This corresponds to the preferred mode frequency. The resulting structures are shown in figures $8(\mathrm{a})$ and (b). It is clear that the difference between the bifurcating and bisecting planes is very weak. In fact, direct numerical simulations of Silva and Métais [3] showed that the efficiency (in terms of increasing the jet spreading rate)

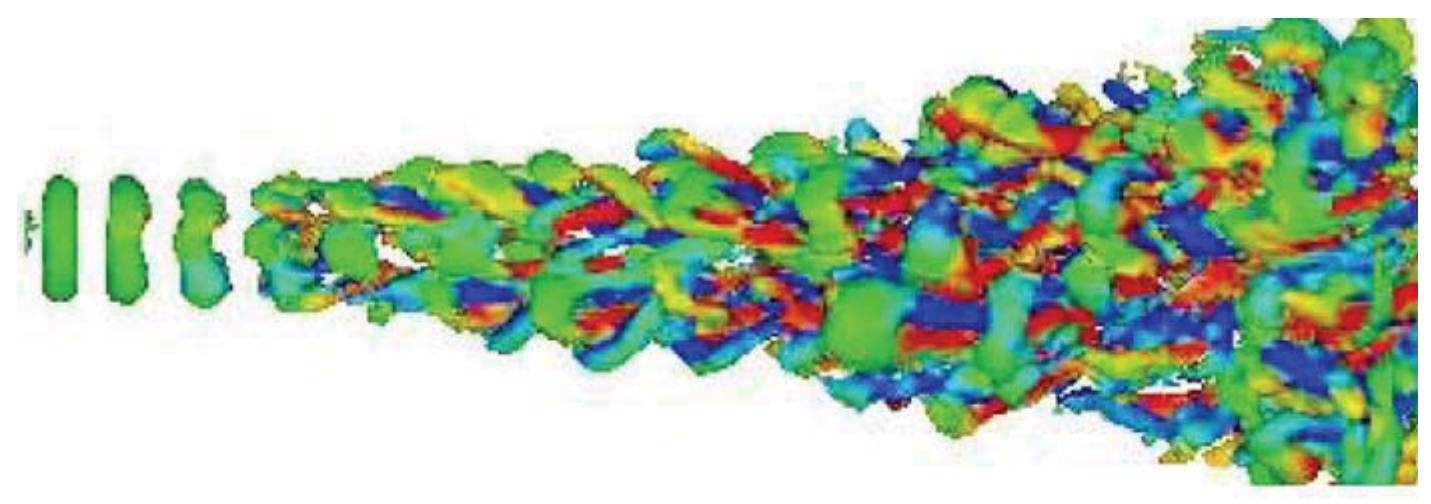

(a)

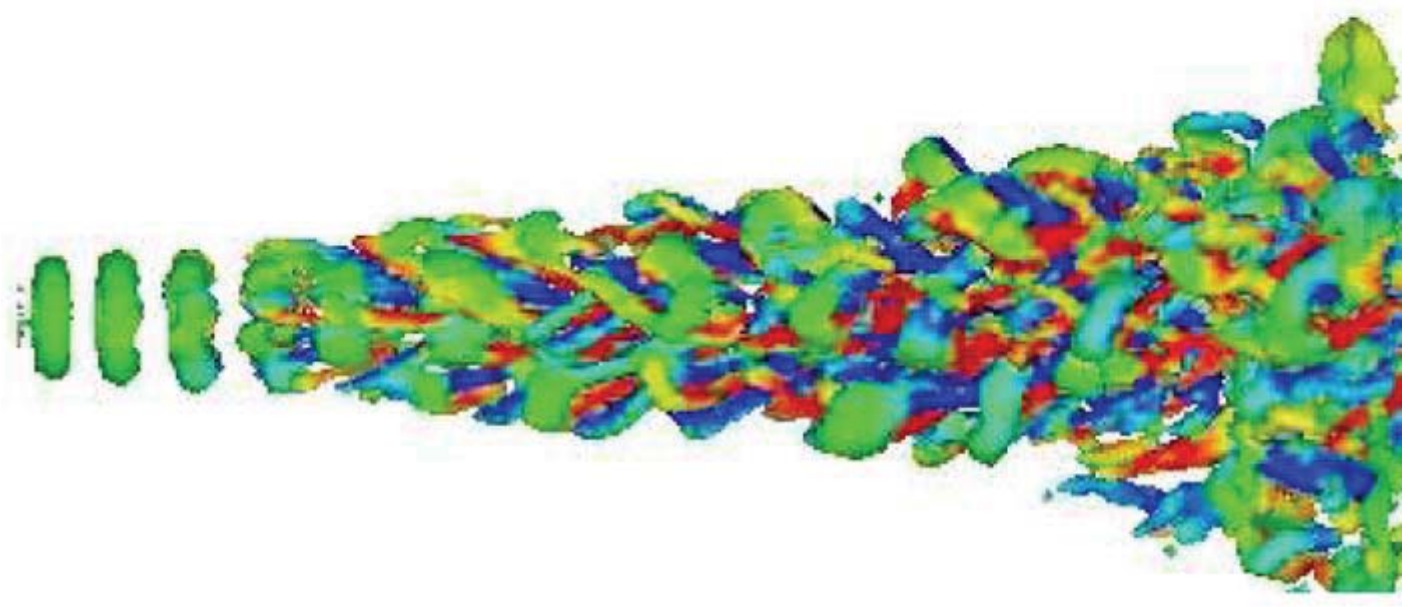

(b)

Figure 8. Isosurfaces of positive $Q$ (green) coloured by the streamwise vorticity $\Omega_{x}$ (red and blue) for the simulation $\mathrm{LES}_{\text {forl }}$. The threshold is $0.20\left(U_{1} / D\right)^{2}$. (a) View in bifurcating plane, (b) view in bisecting plane. 


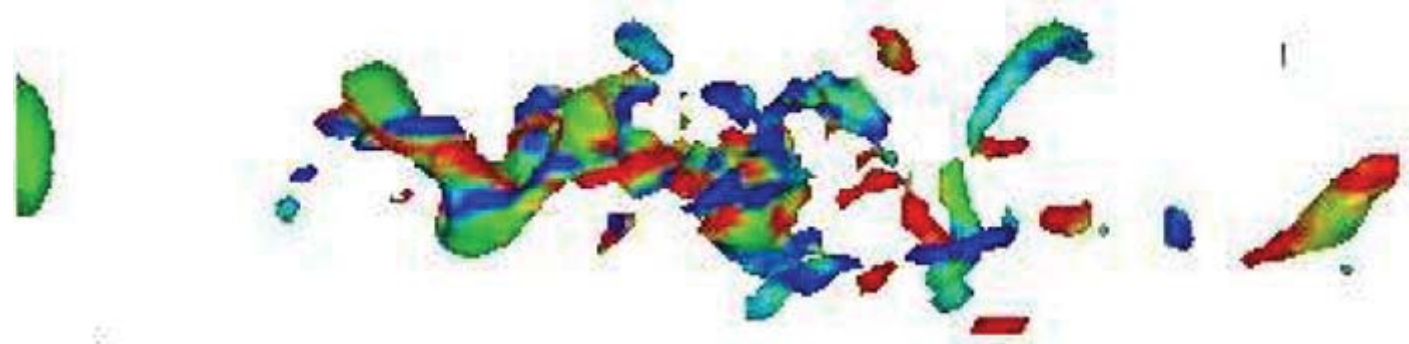

Figure 9. Isosurfaces of low pressure coloured by the streamwise vorticity $\Omega_{x}$ for the simulation LES $\mathrm{for}_{\text {1 }}$. The threshold is $\left(p-p_{0}\right)=-0.05 \rho_{0} U_{1}^{2}$.

of this type of forcing is highly sensitive to the jet Reynolds number. They found that for $R e \geq 5000$, the jet control efficiency is suppressed when using the flapping excitation at the preferred mode frequency, since the bifurcating and bisecting spreading rates are almost identical.

Now we examine the low-pressure isosurfaces in the present simulation for the bisecting plane (see figure 9). One sees with this indicator that the jet flow structures are quickly fragmented at this Reynolds number. This early fragmentation diminishes the efficiency of the flapping excitation to control the local reconnections of toroidal vortex rings, which shows that the present forcing is not capable of creating a bifurcating jet.

To quantify these observations, it is of interest to evaluate certain statistical quantities. Let us look at the shear-layer thicknesses $\delta_{x y}$ in the bifurcating plane and $\delta_{x z}$ in the bisecting plane

$$
\begin{gathered}
\left\langle u_{x}\left(x, y=\delta_{x y}(x), z=0\right)\right\rangle-U_{\operatorname{cof}_{(x y)}}=\frac{\left\langle u_{x}(x, r=0)\right\rangle-U_{2}}{2}, \\
\left\langle u_{x}\left(x, y=0, z=\delta_{x z}(x)\right)\right\rangle-U_{\operatorname{cof}_{(x z)}}=\frac{\left\langle u_{x}(x, r=0)\right\rangle-U_{2}}{2},
\end{gathered}
$$

where $U_{\operatorname{cof}_{(x y)}}=\left\langle u_{x}(x, y=\infty, z=0)\right\rangle$ and $U_{\operatorname{cof}_{(x z)}}=\left\langle u_{x}(x, y=0, z=\infty)\right\rangle$ are the local coflow velocities in the bifurcating and bisecting planes, respectively.

Figure 10(a) shows the downstream evolution of $\delta_{x y}(x)$ and $\delta_{x z}(x)$ for simulation $\mathrm{LES}_{\text {for1 }}$ and for the natural subsonic jet simulation $\left(\operatorname{LES}_{\text {nat1 }}\right)$. One can see that the difference between the bifurcating and bisecting shear-layer thicknesses is limited to the early transitional stages $(x / R \leq 10)$. Further downstream, the bifurcating shear-layer evolution becomes comparable to the bisecting one and to the natural jet.

The downstream evolution of the longitudinal mean centerline velocity (figure 10(b)) confirms these conclusions.

4.2.2 Flapping excitation at the first subharmonic. Since the alternate-pairing vortex organization has been seen to be associated with the growth of subharmonic frequency, it is of interest to excite the jet with the flapping excitation at the first subharmonic frequency. The parameter $\alpha$ in equation (10) is then taken to be equal to 2 .

The following simulation called $\mathrm{LES}_{\text {for } 2}$ is performed with the same Mach and Reynolds numbers as the preceding one (see table 1 for the description of the various runs). The flow visualizations of $\mathrm{LES}_{\text {for2 }}$ simulation can be seen in figures 11(a) and (b). It shows that with this specific subharmonic frequency $f=f_{0} / 2$, the jet-control efficiency is very much improved. Indeed, when compared with natural jet simulation (figure 1), an important jet spreading is obtained in the bifurcating plane, while the jet contracts in the bisecting one. This is confirmed 


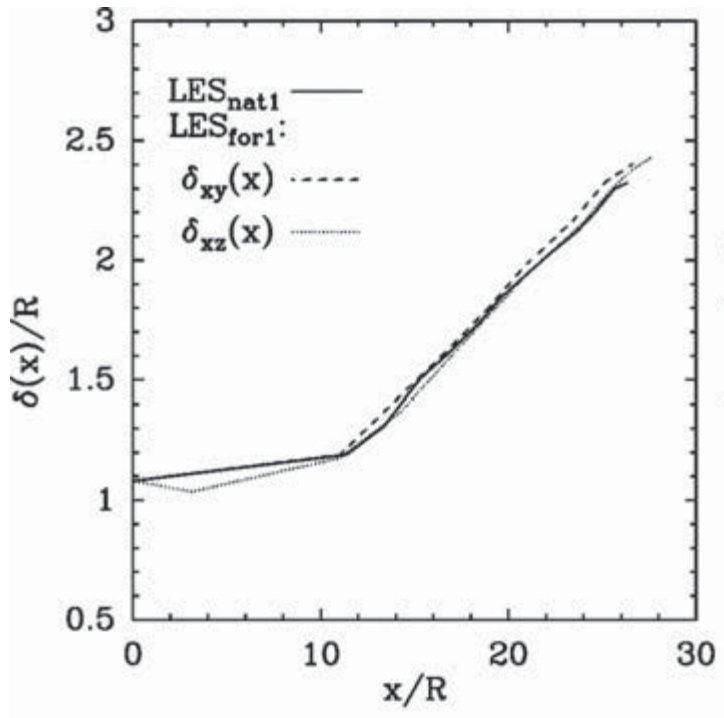

(a)

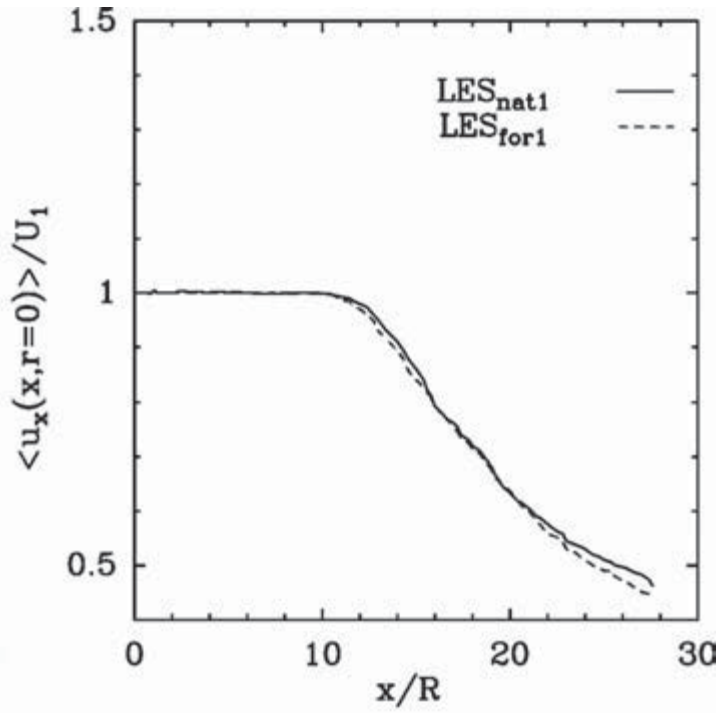

(b)

Figure 10. (a) Downstream evolution of the bifurcating and bisecting shear-layer thicknesses $\left(\delta_{x y}(x)\right.$ and $\left.\delta_{x z}(x)\right)$ for the $\mathrm{LES}_{\text {for1 }}$ simulation; (b) streamwise evolution of the mean centerline velocity. The profiles for the natural jet are also given for comparaison.

by figure 12 representing the downstream evolution of the bifurcating and bisecting shear-layer thicknesses. It turns out that the difference between the bifurcating and bisecting shear-layer thicknesses is huge. Unlike the previous excitation, this difference is now more marked beyond the end of the jet potential core $(x / R>11)$. In agreement with these results, the low-pressure

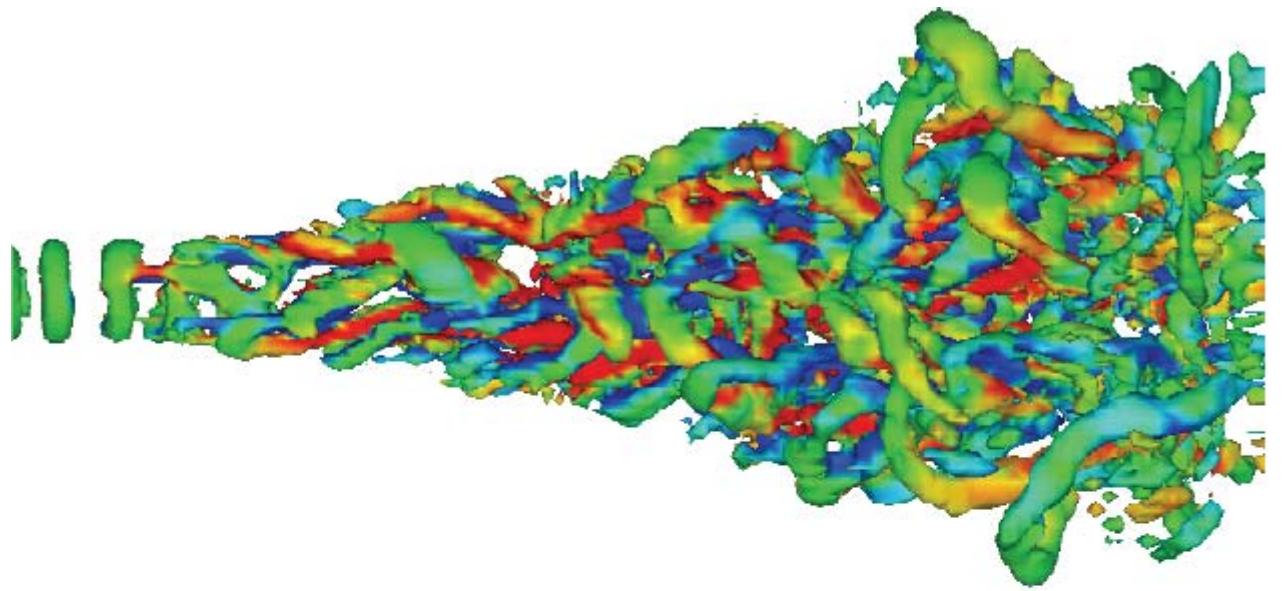

(a)

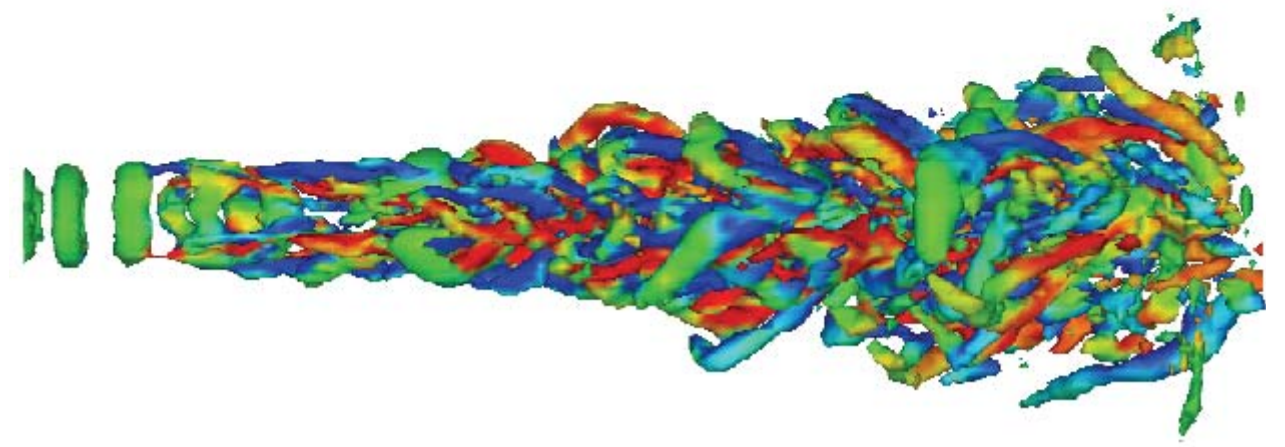

(b)

Figure 11. Isosurfaces of positive $Q$ (green) coloured by the streamwise vorticity $\Omega_{x}$ (red and blue) for the simulation

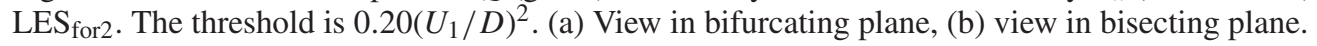




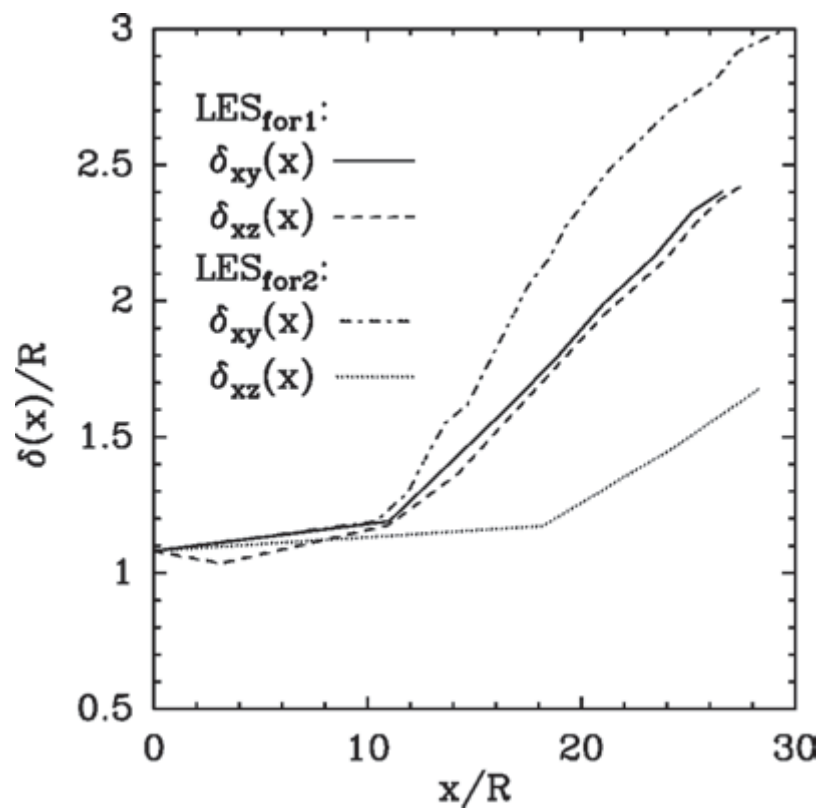

Figure 12. Downstream evolution of the bifurcating and bisecting shear-layer thicknesses $\left(\delta_{x y}(x)\right.$ and $\left.\delta_{x z}(x)\right)$ for the $\mathrm{LES}_{\text {for1 }}$ and $\mathrm{LES}_{\text {for2 }}$ simulations.

isosurfaces in the bisecting plane (see figure 13) show that the large-scale structures survive longer in the present simulation $\left(\mathrm{LES}_{\mathrm{for} 2}\right)$ than in the previous one $\left(\mathrm{LES}_{\mathrm{for} 1}\right)$. In fact, the use of subharmonic frequency ( $f=f_{0} / 2$ ) gives the primary vortices the time to grow naturally before being alternatively confined in the bifurcating plane.

The high spreading rate achieved by the present method of forcing is in good agreement with the experimental results of Suzuki et al. [14] as well as with the direct numerical simulations of Silva and Métais [3] for incompressible round jets. These authors found that the flapping excitation reaches a maximum spreading rate when applied at a frequency corresponding to half the preferred Strouhal number.

In addition to the enhanced jet spreading rate noted in the bifurcating plane, figure 16(a) indicates that the potential core length resulting from the present simulation $\left(\mathrm{LES}_{\text {for } 2}\right)$ is reduced to $13 \%$ with respect to the previous natural jet case (simulation $\operatorname{LES}_{\text {nat } 1}$ ).

4.2.3 Varicose-flapping excitation. Numerous experimental and numerical studies of incompressible round jets $[1,9,36]$ showed that the vortex rings resulting from the upstream varicose excitation are more intense than those obtained in the natural jet case. Thus, we now

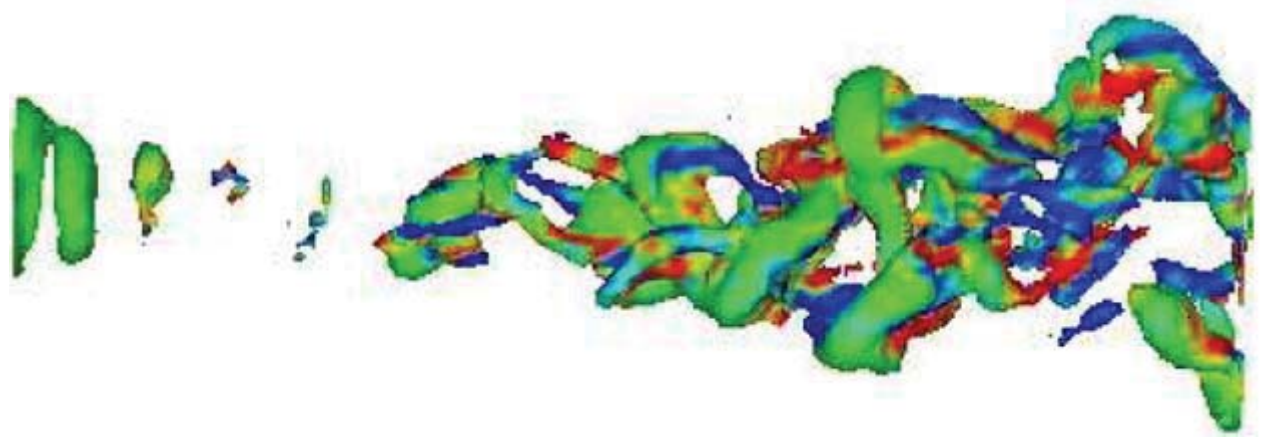

Figure 13. Isosurfaces of low pressure coloured by the streamwise vorticity $\Omega_{x}$ for the simulation LES for 2 . The threshold is $\left(p-p_{0}\right)=-0.05 \rho_{0} U_{1}^{2}$. 


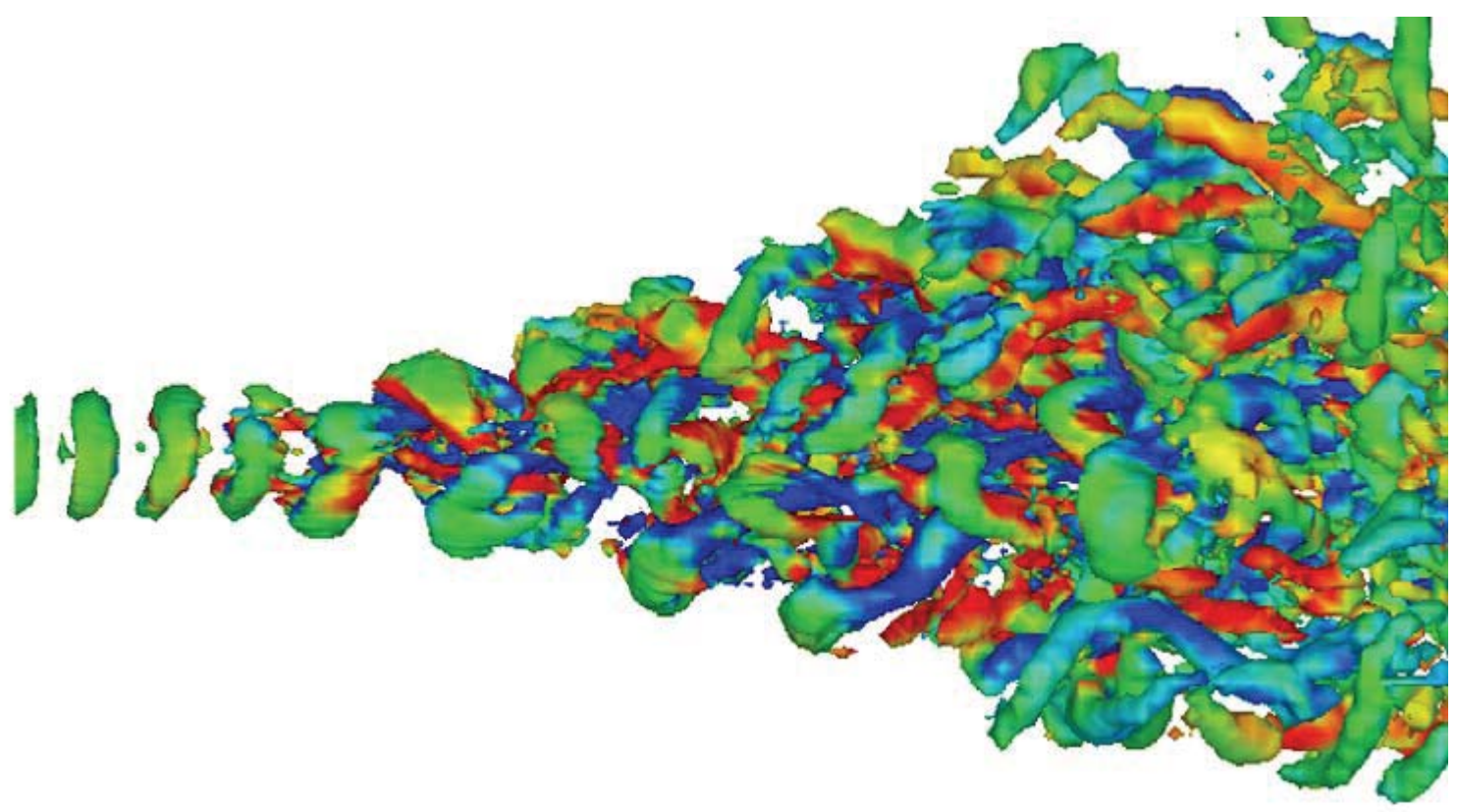

(a)

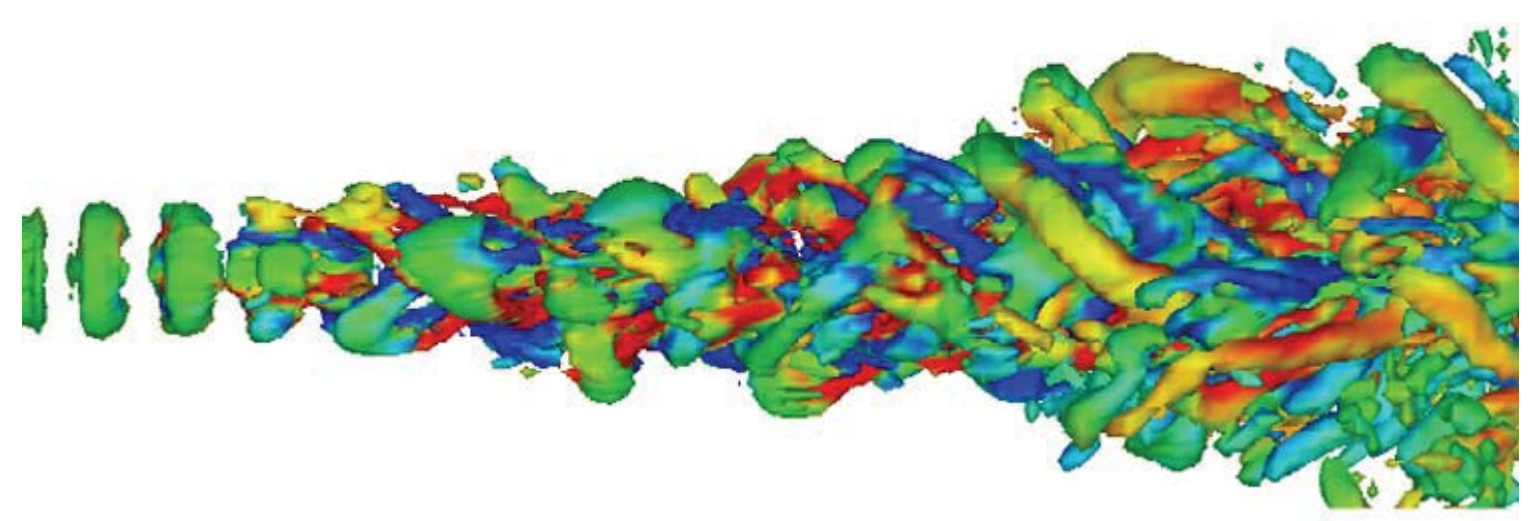

(b)

Figure 14. Isosurfaces of positive $Q$ (green) coloured by the streamwise vorticity $\Omega_{x}$ (red and blue) for the simu-

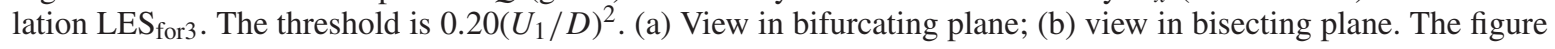
corresponds to animation 1.

superimpose the varicose excitation at the preferred jet frequency to the flapping one at the first subharmonic frequency. Our objective is to increase the strength of primary vortices before throwing them alternatively in the bifurcating plane, in the hope of enhancing the jet spreading rate. The excitation method employed here is similar to that performed by Danaila and Boersma [2], Hilgers [15] and Silva and Métais [3] in DNS and LES of incompressible round jets, respectively. Note that our forcing amplitude is much lower than that used by Danaila and Boersma [2].

The resulting structures are shown in figure 14 and complemented by animation 1 . We can see that now the jet exhibits a spectacular growth in the bifurcating plane. This is confirmed by the spatial evolution of the shear-layer thicknesses in the bifurcating and bisecting planes presented in figure $16(\mathrm{~b})$. The present simulation $\left(\mathrm{LES}_{\text {for3 }}\right)$ yields the highest bifurcating spreading rate when compared with the preceding simulations $\left(\mathrm{LES}_{\text {for1 }}\right.$ and $\left.\mathrm{LES}_{\text {for } 2}\right)$. This enhanced spreading rate in the bifurcating plane is associated with strong positive radial velocities observed in this plane at $x / R=19$ and $x / R=28.5$ (see figure 15). This 


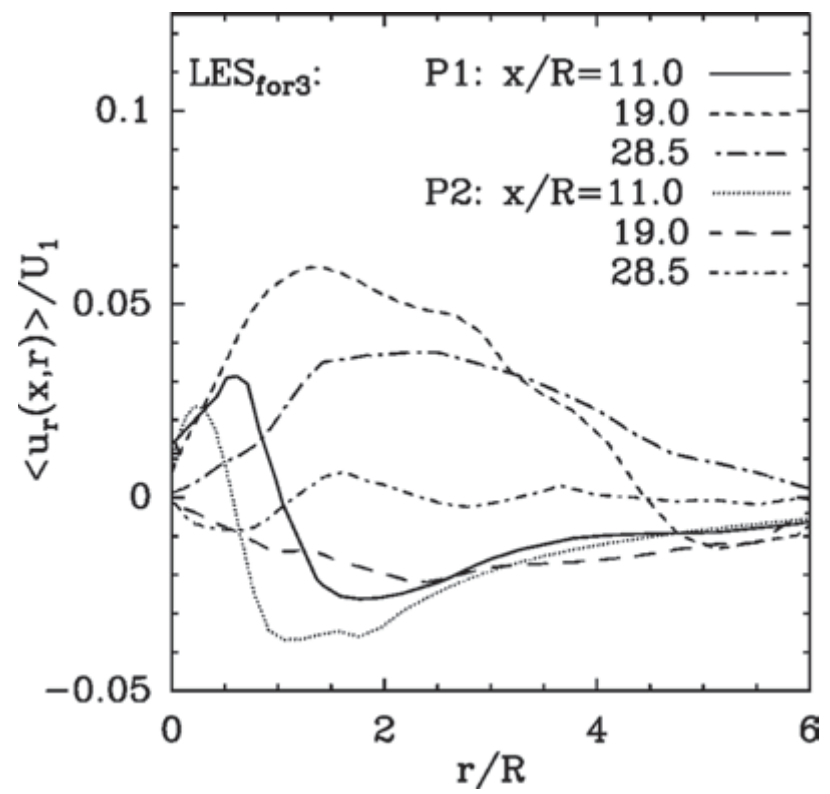

Figure 15. Bifurcating and bisecting profiles of the mean radial velocity for the simulation LES for3. $P_{1}$ and $P_{2}$ represent the bifurcating and bisecting planes, respectively.

suggests that the bifurcating shear-layer grows by radial diffusion inducing an outward fluid motion.

A closer look at figure 14(a) shows that the primary vortex rings keep their coherence until the end of the jet potential core. This observation can be explained by the action of varicose forcing which tends to intensify the primary vortices as mentioned above.

We have evaluated the mean streamwise velocity on the jet centerline, in order to obtain a more quantitative measure of turbulent mixing. We show in figure 16(a) the centerline velocity obtained from different types of forcing and natural jet computations. For the present simulation $\left(\mathrm{LES}_{\mathrm{for} 3}\right)$, the centerline velocity decays faster than in the flapping excitation case

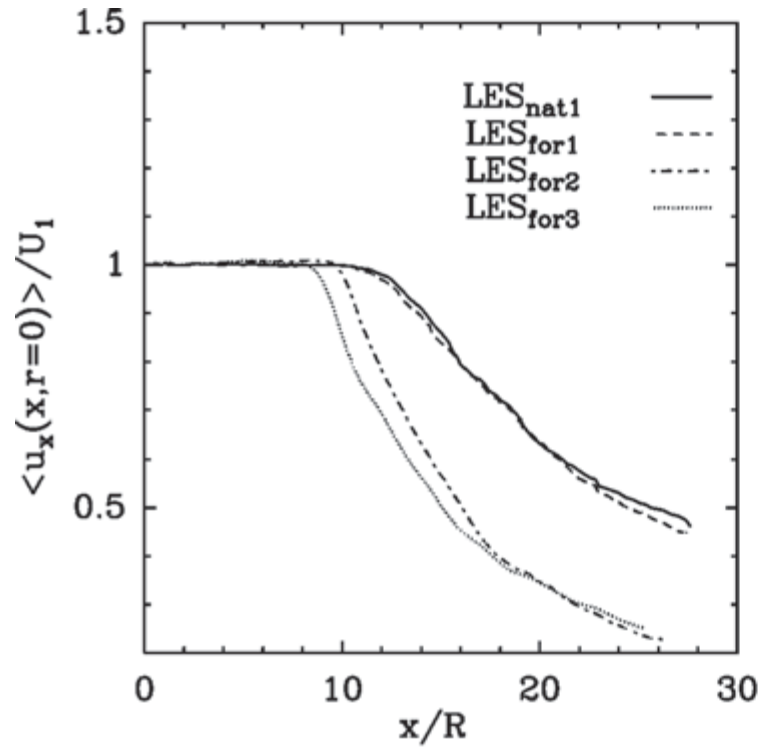

(a)

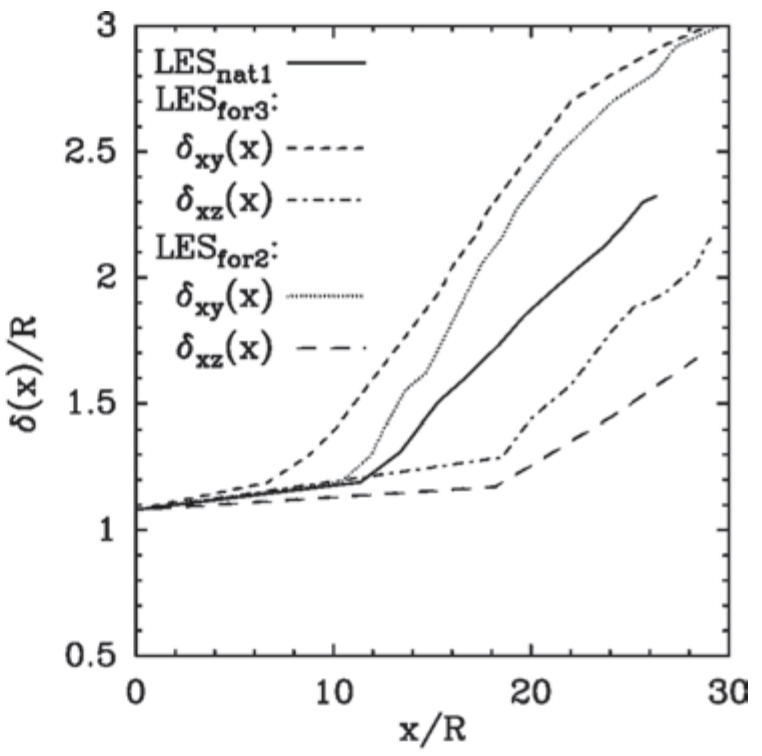

(b)

Figure 16. (a) Streamwise evolution of the mean centerline velocity for the $\mathrm{LES}_{\text {for } 1}$, $\mathrm{LES}_{\text {for2 }}$ and $\mathrm{LES}_{\text {for3 }}$ simulations; (b) downstream evolution of the bifurcating and bisecting shear-layer thicknesses for the $\mathrm{LES}_{\text {for2 }}$ and $\mathrm{LES}_{\text {for3 }}$ simulations. The profiles for the natural jet are also given for comparaison. 
and the natural jet. Indeed, with the varicose-flapping excitation, the centerline velocity starts dropping at about $x / R=8$, representing a decrease of $28 \%$ of the potential core length with respect to the natural jet simulation. This early decay leads to the enhanced mixing rate of the jet which can play a significant role in various industrial applications, among which combustion and aeroacoustics may be mentioned as examples.

These results are in good agreement with experimental studies of Lee and Reynolds [4], Parekh et al. [10], and incompressible DNS of Danaila and Boersma [2] and Silva and Métais [3].

We recall that with this low regime of compressibility $(M=0.7)$, the natural jet simulations exhibit strong similarities with incompressible round jet, as pointed out in section (3).

\subsection{Supersonic jet control}

In this section we are interested in the supersonic jet control using the different types of forcing employed previously in the subsonic jet control, in order to assess their efficiency. The Reynolds number is the same $(R e=36000)$. The preferred jet frequency now corresponds to a Strouhal number $\mathrm{St}_{D}^{0}=0.44$. This value has been evaluated in natural jet simulation (section 3).

4.3.1 Flapping excitation at first subharmonic. The previous study of subsonic jet control showed that the flapping excitation achieves a maximum efficiency at the first subharmonic frequency. We reproduce here such a forcing. In fact, the corresponding simulation $\left(\mathrm{LES}_{\mathrm{for} 4}\right)$ does display a higher jet spreading in the bifurcating plane (see figure 17). In agreement

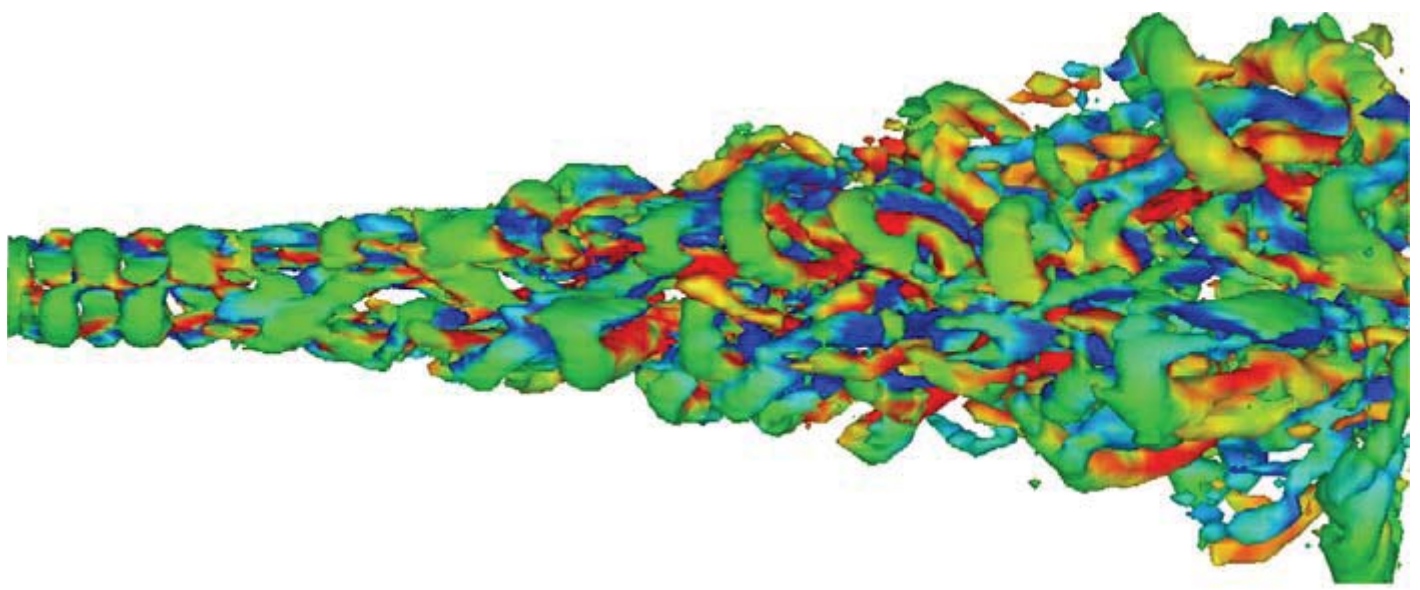

(a)

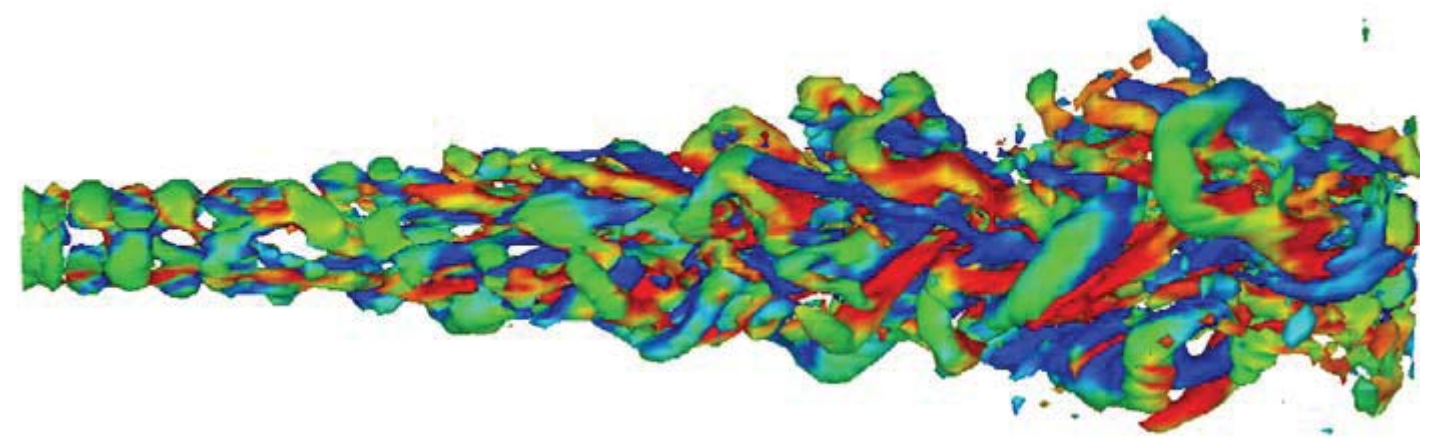

(b)

Figure 17. Isosurfaces of positive $Q$ (green) coloured by the streamwise vorticity $\Omega_{x}$ (red and blue) for the simulation $\mathrm{LES}_{\text {for } 4}$. The threshold is $0.20\left(U_{1} / D\right)^{2}$. (a) View in bifurcating plane, (b) view in bisecting plane. 


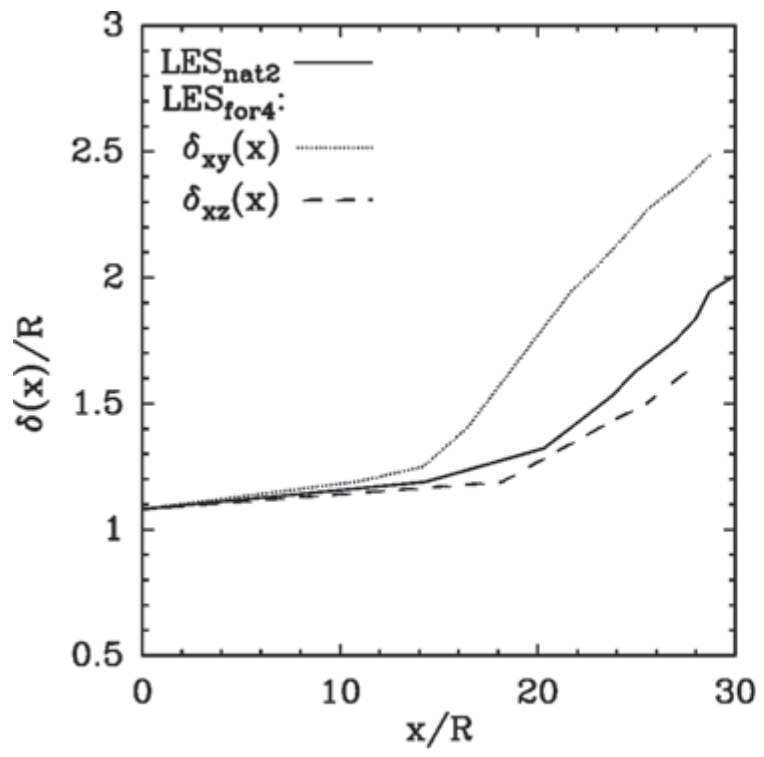

(a)

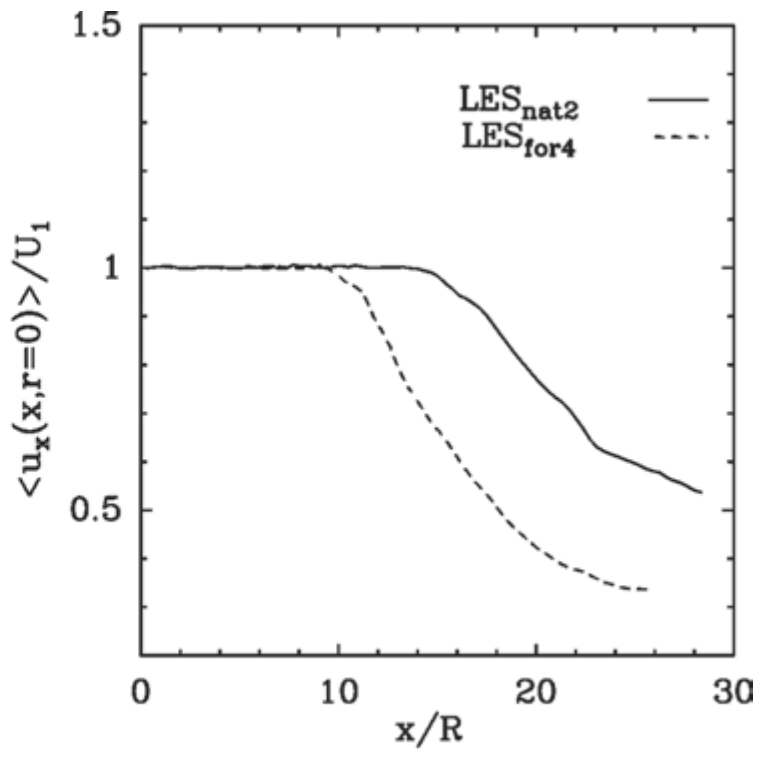

(b)

Figure 18. (a) Downstream evolution of the bifurcating and bisecting shear-layer thicknesses for the simulation $\mathrm{LES}_{\text {for } 4}$, (b) streamwise evolution of the mean centerline velocity for the simulation $\mathrm{LES}_{\text {for4 }}$. The profiles for the natural jet are also given for comparison.

with these visualizations, figure 18(a) shows that the bifurcating shear-layer thickness is much higher than the thickness measured in the bisecting plane and in the natural jet. This difference begins to develop beyond $x / R \approx 10$ inducing a quicker transition to turbulence, which can be seen in figure 18(b). Indeed, the mean centerline velocity decays faster than for the natural jet. In this case, the potential core length is $10 R$ instead of $14 R$, indicating a greater efficiency of this forcing to improve the supersonic jet mixing.

4.3.2 Varicose-flapping excitation. As in the subsonic jet case, we have tested the second type of forcing which combines a varicose excitation at the fundamental and flapping excitation at the first subharmonic. The present simulation is referred to as $\mathrm{LES}_{\text {for5 }}$.

The resulting vortical structures are shown in figure 19. Contrary to what was expected, the jet development is very close to that obtained only with a flapping excitation at first subharmonic. This is confirmed by the downstream evolution of the bifurcating and bisecting shear-layer thicknesses shown in figure 20(a). The difference between the shear-layer evolution for the present simulation and the simulation $\mathrm{LES}_{\mathrm{for} 4}$ is very weak. Moreover, the mean centerline velocity decay is almost similar to that obtained with the flapping excitation $\left(\mathrm{LES}_{\text {for } 4}\right)$.

One possible explanation for the origin of the similarity between the two cases is the weak contribution of the varicose excitation since this later acts principally upon axisymmetric primary vortices. Indeed, as mentioned above for the natural jet, axisymmetric toroidal vortices disappear in supersonic jets, due to an inhibition of Kelvin-Helmhotz instability by compressibility. Note that the present jet visualizations exhibit alternate pairing in the transitional regime downstream of the nozzle.

4.3.3 Flapping excitations at the second subharmonic. We return to the flapping excitation using the second subharmonic frequency. This simulation, referred to as $\left(\mathrm{LES}_{\text {for6 }}\right)$, is carried out with $\alpha$ in equation (10) taken equal to 4 .

The flow visualizations are displayed in figures 21(a) and (b) showing the isosurfaces of positive $Q$ coloured by streamwise vorticity. They are complemented by animation 2 . 


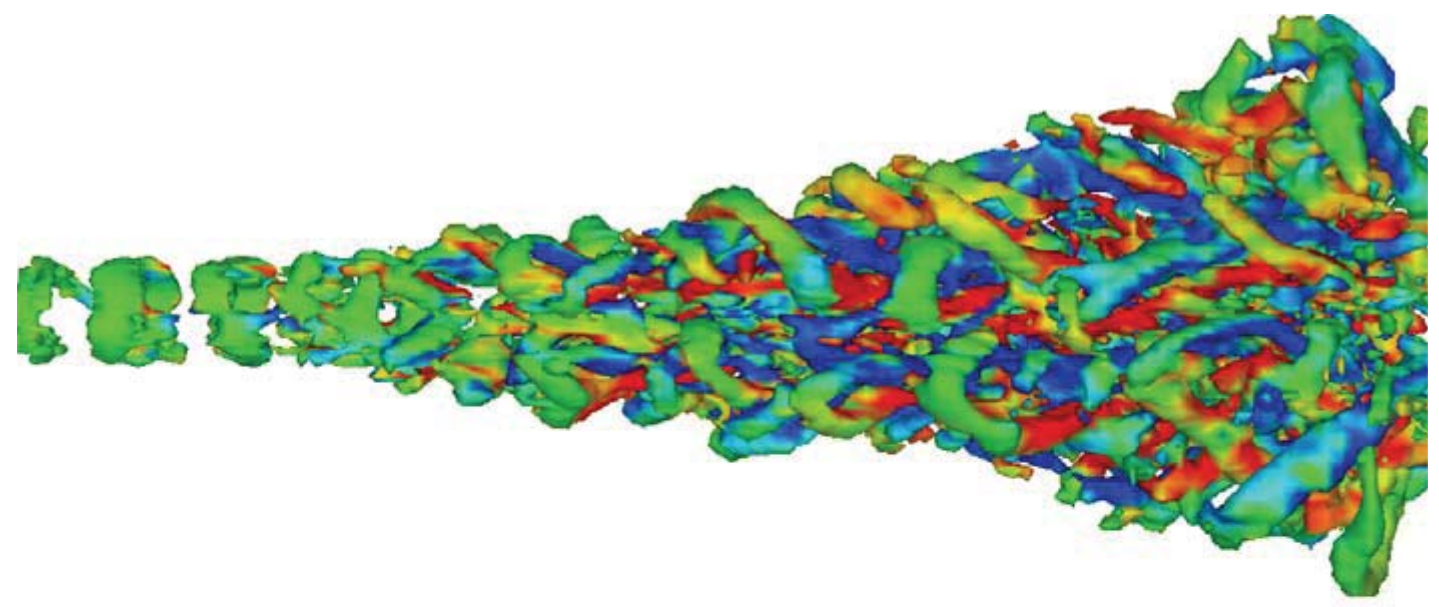

(a)

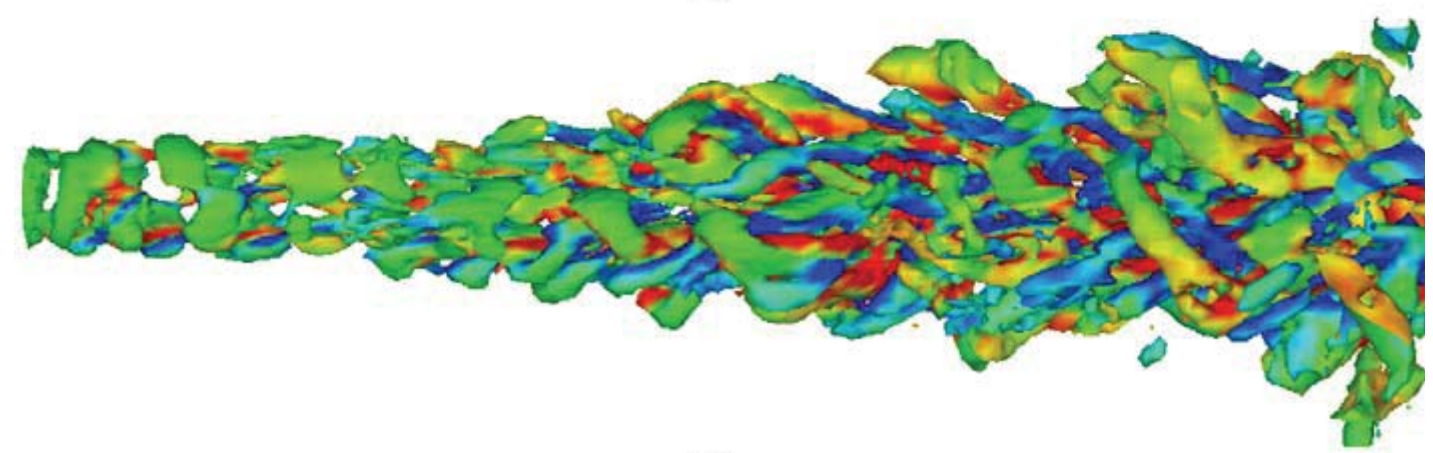

(b)

Figure 19. Isosurfaces of positive $Q$ (green) coloured by the streamwise vorticity $\Omega_{x}$ (red and blue) for the simulation

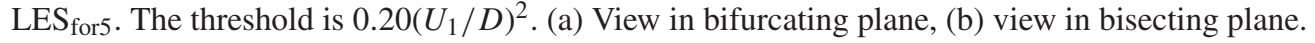

Compared with previous cases of forcing, one can see that the present jet exhibits the highest spreading rate in the bifurcating plane. To confirm these observations, we have examined the downstream evolution of the bifurcating and bisecting shear-layer thicknesses (figure 22(a)) for all the natural and excited jet simulations. In agreement with the visual observations of

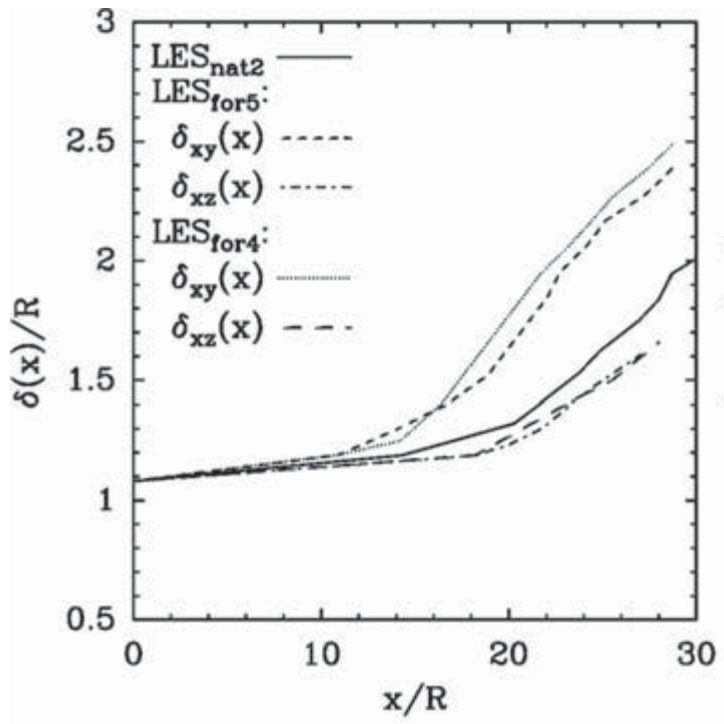

(a)

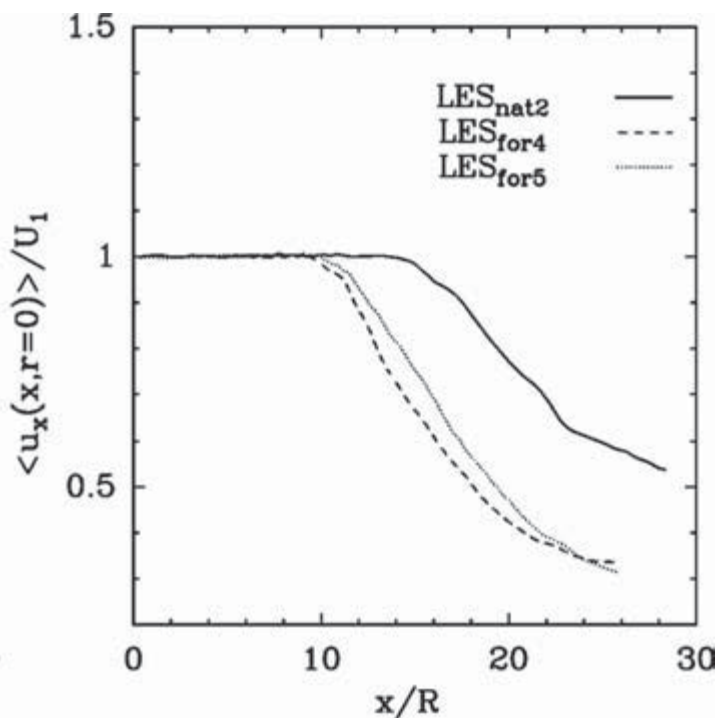

(b)

Figure 20. (a) Downstream evolution of the bifurcating and bisecting shear-layer thicknesses for the LES $_{\text {for } 4}$ and $\mathrm{LES}_{\text {for } 5}$ simulations, (b) streamwise evolution of the mean centerline velocity for the $\mathrm{LES}_{\text {for } 4}$ and $\mathrm{LES}_{\text {for } 5}$ simulations. The profiles for the natural jet are also given for comparison. 


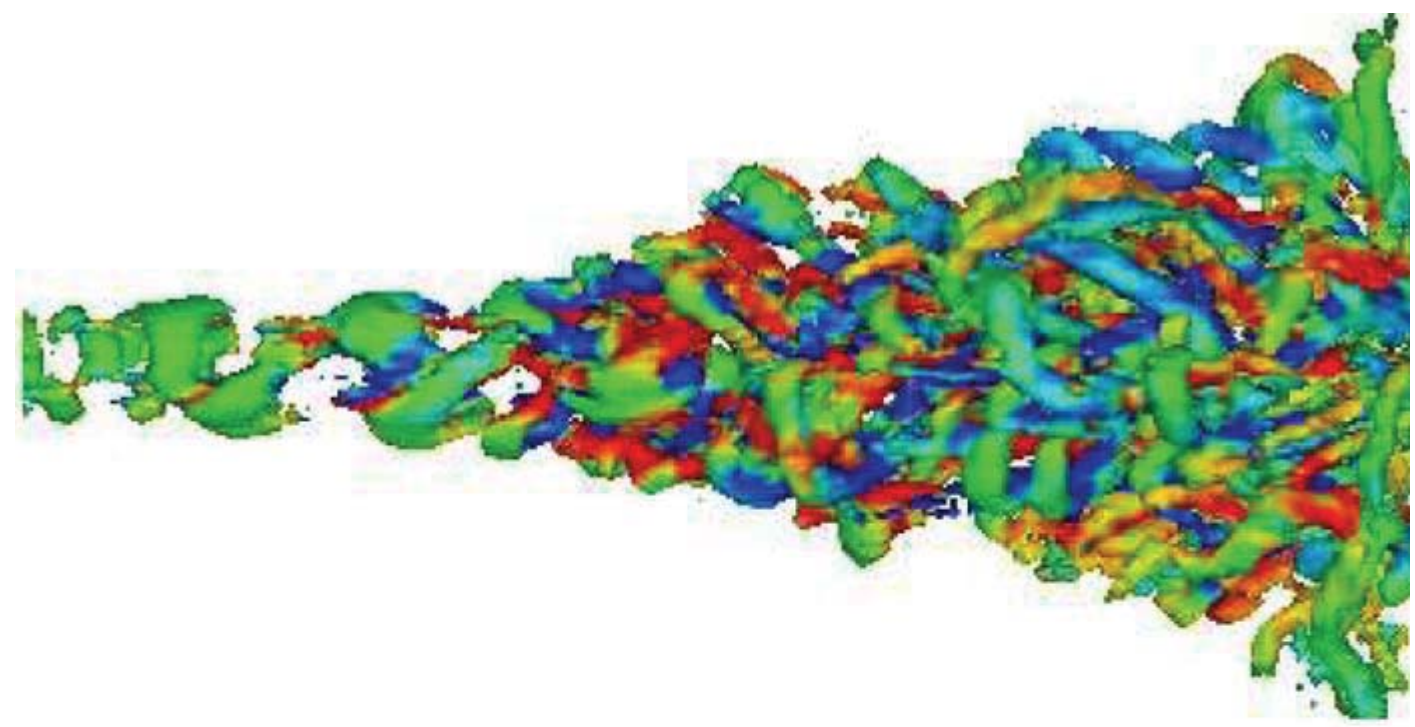

(a)

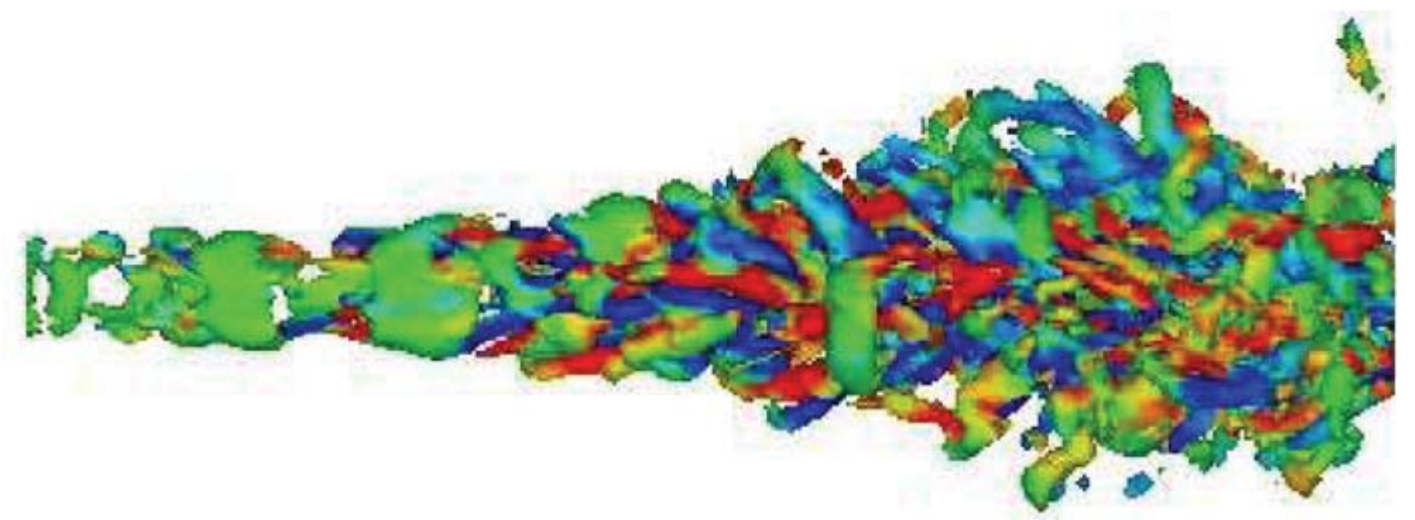

(b)

Figure 21. Isosurfaces of positive $Q$ (green) coloured by the streamwise vorticity $\Omega_{x}$ (red and blue) for the simulation $\mathrm{LES}_{\text {for6 }}$. The threshold is $0.20\left(U_{1} / D\right)^{2}$. (a) View in bifurcating plane, (b) view in bisecting plane. The figure corresponds to animation 2 .

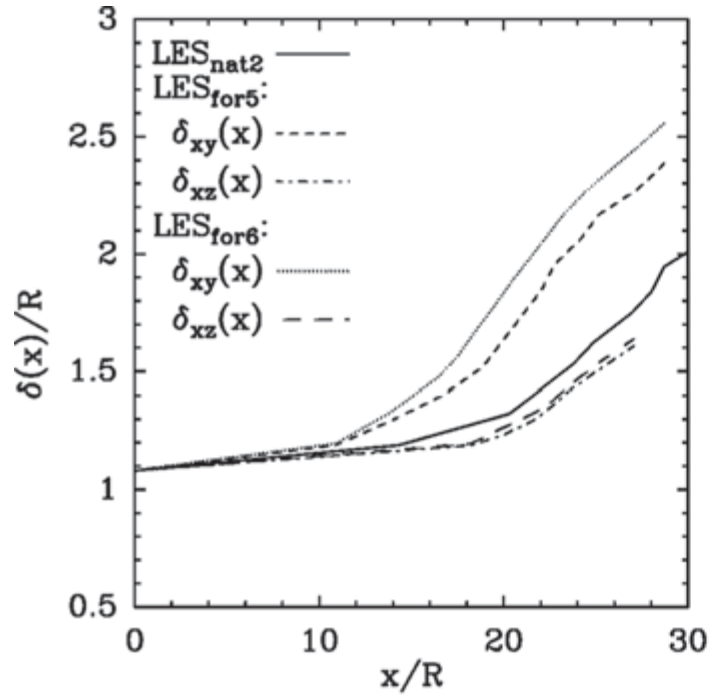

(a)

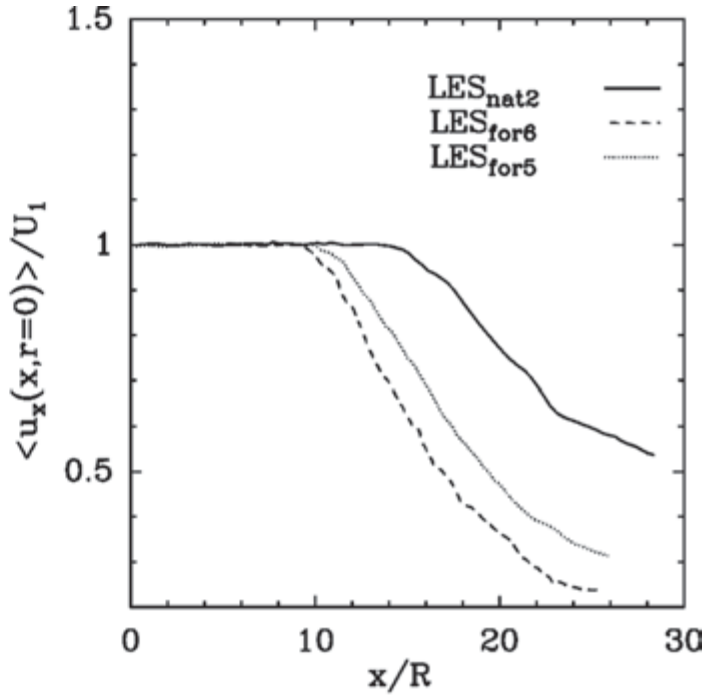

(b)

Figure 22. (a) Downstream evolution of the bifurcating and bisecting shear-layer thicknesses for the $\mathrm{LES}_{\text {for5 }}$ and $\mathrm{LES}_{\text {for6 }}$ simulations, (b) streamwise evolution of the mean centerline velocity for the $\mathrm{LES}_{\text {for5 }}$ and $\mathrm{LES}_{\text {for6 }}$ simulations. The profiles for the natural jet are also given for comparaison. 
figure 21, figure 22(a) shows that the present excitation leads to the highest spreading rate in the bifurcating plane, while in the bisecting plane the jet spreading is shown to be almost similar to the one obtained with the preceding forcing case.

The streamwise evolution of the mean centerline velocity is presented in figure 22(b). We include for comparison the mean centerline velocity profiles obtained for all the natural and excited supersonic jet simulations. We show that the mean centerline velocity decays faster in the present case than in the previous natural and forced supersonic jet simulations. Indeed, the centerline velocity now starts dropping near $x / R=10$, representing an important decrease of $28 \%$ with respect to the natural jet.

These results highlight that with a suitable forcing frequency, the flapping excitation strategy is efficiently able to control the supersonic jet dynamics.

\section{Conclusion}

We have described in this paper the different forcing strategies aimed to control the spatial evolution of the compressible subsonic and supersonic round jets with the aid of large-eddy simulation. Our goal was to find upstream perturbations which maximize mixing in jets using information provided by a LES of natural compressible round jets. The simulated jet is initiated upstream by a basic velocity profile close to a top-hat shape, which is perturbed by various small forcings.

Our LES of natural jets have been carried out at a Reynolds number $R e=36000$ and for two Mach numbers: Mach 0.7 and Mach 1.4 for subsonic and supersonic cases, respectively. At this stage, the upstream velocity profile is forced by a weak random isotropic perturbation.

Visualizations of the subsonic jet simulation exhibit the shedding of axisymmetric vortex rings close to the numerical nozzle. Their passage frequency at the end of the potential core corresponds to the preferred Strouhal number $\mathrm{St}_{D}^{0}=0.39$. Further downstream, the rings give rise to a vortex arrangement consisting in alternate localized pairings which cause an important jet spreading.

Two types of upstream perturbations have been considered here for controlling the jet dynamics. The first, called flapping excitation, is similar to the excitation proposed by Urbin and Métais [1], and used by Danaila and Boersma [2] and Silva and Métais [3]. The second type of excitation is of the varicose-flapping type and consists in the combination of fundamental varicose and subharmonic flapping perturbations, similar to that used by Danaila and Boersma [2] and Silva and Métais [3] in their incompressible simulations. For both subsonic and supersonic cases, we have analysed the jet response to each forcing method using several forcing frequencies.

In the subsonic jet case, the highest spreading rate is obtained with the varicose-flapping excitation. We have also checked that the potential core length is then reduced by $27 \%$ with respect to the natural jet simulation. These results have analogies with the experimental results of Lee and Reynolds [4], Parekh et al. [10] and incompressible DNS or LES of Danaila and Boersma [2] and Silva and Métais [3].

In the supersonic jet case, the most efficient strategy for jet control, as far as a high spreading is concerned, is obtained by using a flapping excitation at the second subharmonic frequency. The potential core length is now reduced by $28 \%$ with respect to the natural jet simulation. This causes a quick transition to turbulence and leads to the more enhanced mixing rate of the jet which can play a significant role in various industrial applications such as combustion and aeroacoustics.

Future work will concern the study of acoustic fields resulting from each excitation type in order to evaluate the ability of these different forcings to reduce the jet acoustic emission. 


\section{References}

[1] Urbin, G. and Métais O., 1997, Large-eddy simulations of three-dimensional spatially-developing round jets. In Direct and large-eddy simulations II, P.R. Chollet, J.P. Voke and L. Kleiser, Kluwer: Dordrecht, pp. 539-542.

[2] Danaila, I. and Boersma, B.J., 2000, Direct numerical simulation of bifurcating jets. Physics of Fluids A, 12 , $1255-1257$.

[3] da Silva, C.B. and Métais, O., 2002, Vortex control of bifurcating jets: a numerical study. Physics of Fluids, 14, 3798-3819.

[4] Lee, M. and Reynolds, W.C., 1985, Bifurcating and blooming jets at high Reynolds number. 5th Symposium on Turbulent Shear Flows, New York.

[5] Bishop, K.A., Ffowcs Williams, J.E. and Smith, W., 1971, On the noise sources of the unsuppressed high-speed jet. Journal of Fluid Mechanics, 50, 21-31.

[6] Juvé, D., Sunyach, M. and Comte-Bellot, G., 1980, Intermittency of the noise emission in subsonic cold jets. Journal of Sound and Vibration, 71(3), 319-332.

[7] Laufer, J. and Yen, T., 1983, Noise generation by a low-Mach-number jet. Journal of Fluid Mechanics, 134, $1-31$.

[8] Gutmark, E.J. and Grinstein, F.F., 1999, Flow control with noncircular jets. Annual Review of Fluid Mechanics, 31, 239-272.

[9] Crow, S.C. and Champagne, F.H., 1971, Orderly structure in jet turbulence. Journal of Fluid Mechanics, 48, 547-591.

[10] Parekh, D., Leonard, A., Reynolds, W.C. and Mungal, M.G., 1987, Bifurcating of round air jets by dual-mode acoustic excitation. AIAA Paper 87-0164.

[11] Corke, T.C. and Kusec, S.M., 1993, Resonance in axisymmetric jets with controlled helical-mode input. Journal of Fluid Mechanics, 249, 307-336.

[12] Longmire, E.K. and Duong, L.H., 1995, Bifurcating jets generated with stepped and sawtooth nozzles. Physics of Fluids A, 8, 978-992.

[13] Parekh, D., Glezer, V., Wiltse, J.M. and Smith, D.M., 1996, Experimental study of compressible turbulent mixing layers. AIAA Paper 96-0308.

[14] Suzuki, H., Kasagi, N. and Suzuki, Y., 2000, Active control of an axisymmetric jet with an intelligent nozzle. 1st Turbulent Shear Flow Phenomena.

[15] Hilgers, A., 2000, Control and optimization of turbulent jet mixing. Annual Research Briefs, Center for Turbulence Research, Stanford University (Stanford).

[16] Hussein, H.J., Capp, S.P. and George, W.K., 1994, Velocity measurements in a high-Reynolds-number momentum-conserving, axisymmetric, turbulent jet. Journal of Fluid Mechanics, 258, 31-75.

[17] Zaman, K.B.M.Q. and Hussain, K.M.F., 1980, Vortex pairing in a circular jet under controlled excitation. Part 1. General jet response. Journal of Fluid Mechanics, 101, 449-491.

[18] Maidi, M. and Lesieur, M., 2005, Large-eddy simulations of spatially-growing subsonic and supersonic turbulent round jets. Journal of Turbulence, 6(38), 1-20.

[19] Mac Cormack, R.W., 1969, The effects of viscosity in hypervelocity impact cratering. AIAA Paper, 69-354.

[20] Gottlieb, D. and Turkel, E., 1976, Dissipative two-four methods for time-dependent problems. Journal of Computational Physics, 30, 703-723.

[21] Normand, X. and Lesieur, M., 1992, Direct and large-eddy simulations of transition in the compressible boundary layer. Theoritical and Computational Fluid Dynamics, 3, 231-252.

[22] Maidi, M., 2004, Étude et contrôle des jets ronds compressibles par simulations des grandes échelles. PhD Thesis, Grenoble.

[23] Poinsot, T.J. and Lele, S.K., 1992, Boundary conditions for direct simulations of compressible viscous flows. Journal of Computational Physics, 101, 104-129.

[24] Sandhu, H.S., Sandham, N.D., 1994, Boundary conditions for spatially growing compressible shear layers. Tech. Rep., Department of Aeronautical Engineering, Queen Mary \& Westfield College.

[25] Michalke, A. and Hermann, G., 1982, On the inviscid instability of a circular jet with external flow. Journal of Fluid Mechanics, 114, 343-359.

[26] Freymuth, P., 1966, On transition in a separated laminar boundary layer. Journal of Fluid Mechanics, 25, 683-704

[27] Métais, O. and Lesieur, M., 1992, Spectral large-eddy simulation of isotropic and stably stratified turbulence. Journal of Fluid Mechanics, 239, 157-194.

[28] Ducros, F., Comte, P. and Lesieur, M., 1996, Large-eddy simulation of transition to turbulence in a boundary layer developing spatially over a flat plate. Journal of Fluid Mechanics, 326, 1-36.

[29] Hunt, J.C.R., Wray, A.A. and Moin, P., 1988, Eddies, stream, and convergence zones in turbulent flows. Annual Research Briefs, Center for Turbulence Research, Stanford University (Stanford).

[30] Dubief, Y. and Delcayre, F., 2000, On coherent-vortex identification in turbulence. Journal of Turbulence, 1(11), 1-22.

[31] Broze, G. and Hussain, F., 1996, Transition to chaos in a forced jet: intermitency, tangent bifurcations and hysteresis. Journal of Fluid Mechanics, 311, 37-71.

[32] Clemens, N.T. and Mungal, M.G., 1995, Large-scale structure and entrainment in the supersonic mixing layer. Journal of Fluid Mechanics, 284, 171-216. 
[33] Thurow, B., Samimy, M. and Lempert, W., 2003, Compressibility effects on turbulence structures of axisymmetric mixing layers. Physics Fluids, 15, 1755-1765.

[34] Hussain, K.M.F. and Zaman, K.B.M.Q., 1981, Preferred mode of the axisymmetric jet. Journal of Fluid Mechanics, 110, 39-71.

[35] Petersen, R.A., 1978, Influence of wave dispersion on vortex pairing in a jet. Journal of Fluid Mechanics, 89, 469-495.

[36] Martin, J.E. and Meiburg, E., 1991, Numerical investigation of the three-dimensionaly evolving jets subject to axissymmetric and azimutal perturbations. Journal of Fluid Mechanics, 230, 271-318.

[37] Hilgers, A. and Boersma, B.J., 2001, Optimization of turbulent jet mixing. Fluid Dynamics Research, 29, 345-368. 